\title{
A feasibility study of a cross-diagnostic, CBT-based psychological intervention for acute mental health inpatients: Results, challenges and methodological implications
}

Running Head: Psychologically informed acute inpatient care: A feasibility trial

\author{
Charlotte Paterson ${ }^{\mathrm{a}}$ \\ Thanos Karatzias ${ }^{\mathrm{a}}$ \\ Sean Harper ${ }^{\mathrm{b}}$ \\ Nadine Dougall ${ }^{\mathrm{a}}$ \\ Adele Dickson $^{\mathrm{c}}$ \\ Paul Hutton ${ }^{\mathrm{a}}$
}

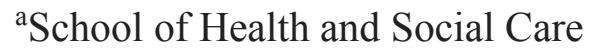

Edinburgh Napier University, 9 Sighthill Court, Edinburgh, EH11 4BN, Scotland.

bPsychology Department

Royal Edinburgh Hospital, NHS Lothian, Tipperlinn Road, Edinburgh, EH10 5HF.

${ }^{\mathrm{c}}$ Department of Psychology and Allied Health Sciences

Glasgow Caledonian University, Cowcaddens Road, Glasgow, G4 0BA, Scotland.

Corresponding author: Charlotte Paterson;

Email: c.paterson@napier.ac.uk

Address: School of Health and Social Care, Edinburgh Napier University, 9 Sighthill Court, Edinburgh, EH11 4BN.

\section{Declaration of interest}

There was no funding for this work. $\mathrm{CP}, \mathrm{TK}, \mathrm{SH}, \mathrm{ND}$ and $\mathrm{AD}$ report no financial conflicts of interests. PH has been a co-investigator on NIHR-funded trials of psychological therapy for people with psychosis, and is a member of the National Institute for Health and Care Excellence Committee which is developing new guidance on supporting decision-making and mental capacity (Decision-Making and Mental Capacity; GID-NG10009). 


\begin{abstract}
Objectives

Acute psychiatric inpatient wards are characterised by minimal provision of therapeutic activities and high readmission rates. Implementation of a comprehensive inpatient psychological intervention service has been recommended to overcome these problems, however whether this is feasible or effective remains unclear.
\end{abstract}

\title{
Methods
}

This non-randomised parallel cluster feasibility trial examined the feasibility of delivering and evaluating cross-diagnostic psychologically informed acute psychiatric care (the E-Acute Psychological Inpatient Therapy Service; EDAPTS), and gathered preliminary clinical outcome data. Patients able to consent and complete questionnaires were recruited from two adult acute wards (i.e. clusters) and received either EDAPTS plus TAU or TAU.

\section{Results}

Between October 2015 and 2016, 96 inpatients were recruited. Findings suggested there were good data completion rates for several clinical outcomes, that several EDAPTS components were successfully delivered and that some initial effects appeared to favour the intervention, depending on outcome. However difficulties relating to the recruitment process were also identified, as well as problems relating to adequate delivery of group therapies, participant engagement in some intervention components, and data-completion at follow-up.

\section{Conclusion}

These issues, and the feasibility of randomisation and rater-blinding, have important implications for the design of future trials. Overall, this study provides an important insight into 
the challenges and complexities of developing and evaluating a comprehensive psychological intervention service in an acute psychiatric setting.

\section{Practitioners points}

- Individual therapy sessions can be delivered in the acute environment.

- The EDAPTS intervention showed some promise on outcomes of distress and selfefficacy.

- Delivery of nurse-led groups was challenging and may need to be embedded into routine clinical practice to increase intervention and outcome reach.

- More parameters, e.g. randomisation at cluster level, should be tested before progressing to an adequately powered, single-blind, definitive cluster RCT.

\section{Funding}

This research did not receive any specific grant from funding agencies in the public, commercial, or not-for-profit sectors. 


\section{Introduction}

Acute mental health inpatient services have been criticised for being nontherapeutic (Schizophrenia Commission, 2012) and lacking in therapeutic activities and appropriate interventions (British Psychological Society, 2015; Janner, 2007; Mind, 2004). As a possible consequence of this, a high proportion of patients are readmitted (Care Quality Commission, 2015; Information Services Division Scotland, 2012). In response, clinicians and regulatory bodies have suggested that providing intensive psychological interventions during acute admissions may improve outcomes (British Psychological Society, 2012; Janner, 2007; Veltro et al., 2006). This is partly due to the growing evidence base supporting the effectiveness of cognitive behavioural therapy (CBT), and other psychological therapies, for people with severe mental illness such as psychosis (Jauhar et al., 2014; Turner, Van Der Gaag, Karyotaki, \& Cuijpers, 2014; Wykes, Steel, Everitt, \& Tarrier, 2008). Some psychological interventions have been adapted for acute inpatient settings, and meta-analysis has shown that, compared to control, they are associated with reductions in emotional distress (depression and anxiety) and readmissions, respectively (Paterson, et al., 2018). However, this research has predominantly focused on therapies that target specific diagnoses, making it unclear whether it can be generalised to routine acute inpatient care, where diagnostic heterogeneity is the norm.

Although not routinely offered, some cross-diagnostic psychosocial initiatives have been developed for acute inpatient settings: the Tidal Model and the Refocusing Model. These initiatives aim to improve ward milieu and the quality of staff-patient interactions, regardless of diagnosis. Preliminary findings suggest their implementation is associated with improvements in ward incidents, length of stay, staff sickness (Dodds \& Bowles, 2001; Gordon, 2005) and patient outcomes (Stevenson, Barker, \& Fletcher, 2002). More recently, 
psychological therapies, such as CBT-based interventions, have also been adapted for acute inpatients, again regardless of diagnosis (Clarke \& Wilson, 2009; Clarke \& Nicholls, 2018). Although they appear to be acceptable to staff and patients (Araci \& Clarke, 2016), and are associated with improvements in self-efficacy, confidence in expressing emotions, perceived locus of control ( $\mathrm{n}=14)$ (Durrant et al., 2007) and distress ( $\mathrm{n}=131)$ (Araci \& Clarke, 2016), these interventions have not been compared to an appropriate control condition. This makes is unclear whether the observed benefits are attributable to the intervention, natural recovery or regression to the mean. It is also unclear whether these interventions can safely reduce rates of readmission which is unfortunate given the high proportion of patients readmitted to acute inpatient services (Care Quality Commission, 2015; Information Services Division Scotland, 2012), the associated cost, and the increased risk of suicide during and after inpatient care (Bickley et al., 2013).

A definitive evaluation of the effect of an acute psychological inpatient therapy service on readmission and other important outcomes requires a well-powered cluster randomised controlled trial. However such a trial would be large, highly expensive and time-consuming, meaning evidence of proof-of-concept, feasibility and acceptability is first required before they can be undertaken (Campbell et al., 2000). The aim of this study was to examine these questions during evaluation of the E-Acute Psychological Inpatient Therapy Service (EDAPTS), as well as report preliminary data on its potential effects on key outcomes of interest to patients and providers, including readmission and safety, when compared to acute psychiatric care alone (treatment as usual; TAU).

\section{Method}




\section{Ethical approval and trial registration}

The study was approved by NHS Research Ethics Committee, and the protocol was registered online. Post-registration changes are detailed in Appendix A, and discussed further below.

\section{Design}

Our initial aim was to establish whether EDAPTS plus TAU is more effective than TAU for the outcomes of readmission, symptoms and psychological distress. A non-randomised comparison of an EDAPTS ward and a TAU ward was therefore designed and implemented to address these questions, and inform the development of a larger scale RCT. However recruitment difficulties and other implementation-related challenges necessitated further feasibility work first, and the trial was refocused on addressing these issues and testing other trial parameters (Eldridge, Lancaster, et al., 2016). Accordingly, this study is most appropriately described as a non-randomised parallel feasibility trial using all trial processes (except randomisation and assessor blinding), as a miniature of a future definitive cluster trial, and has been reported in accordance with CONSORT guidelines (Eldridge et al., 2016).

\section{Outcomes}

\section{Primary outcomes}

Our revised primary objective was to establish the feasibility of delivering and evaluating EDAPTS in an acute inpatient psychiatric care setting in preparation for a definitive trial. This was evaluated using criteria developed by Shanyinde et al. (2011) to inform decision making after a pilot or feasibility study (Bugge et al., 2013; Craig et al., 2013). These criteria also map onto stage one of the ADePT (A process for Decision-making after Pilot and feasibility Trials) process (Bugge et al., 2013). Thus, primary outcomes of interest were as follows: 
a. Eligibility rate: the proportion of those who were screened to participate as a percentage of those eligible to participate (i.e. fulfilled eligibility criteria of the study).

b. Consent rate: the willingness of wards to participate and the proportion of those who consented to participate as a percentage of those who were approached to participate.

c. Completion rate: a) Proportion of clinical outcome questionnaires (CORE-10, BSI-18 and MHCS) completed at each time point (baseline, post-intervention and follow-up) as a percentage of those who entered the trial, with reasons for attrition where possible and assumed missing data mechanisms. This also provided an inverted measure of trial attrition.

d. Proportion of readmission data successfully collected as a proportion of those who entered the trial, with reasons for missingness were possible and assumed missing data mechanism.

e. Number of EDAPTS components delivered.

f. Number of sessions (of each EDAPTS component) delivered.

g. Average number of overall, group and individual sessions received by participants

h. Number and proportion of participants in the intervention group who engaged in EDAPTS.

i. Number and proportion of participants who did not engage (with reasons where possible).

We did not formally measure acceptability, and we did not conduct an economic evaluation. Because the trial took place in one site, we also could not assess the logistical demands which may be involved in a multi-site trial. 


\section{Secondary outcomes}

Following our change in objectives, gathering clinical outcome data became a secondary aim. This was used to inform a future definitive trial, for which the planned primary outcome was number of readmissions at 12-month follow-up, however readmissions at 6-months were assessed in the current trial to indicate direction of effect within the available timescale. Secondary clinical outcomes for a future trial are described below and were collected at preintervention, post-intervention and 6-months after discharge:

a) The Brief Symptom Inventory (BSI) 18 (Derogatis, 2001): an 18-item self-report measure of psychological distress including domains of somatization, anxiety and depression. Each item is scored on a 5 point Likert scale ( $0=$ 'not at all' to $4=$ 'extremely') with a higher score indicating higher severity.

b) The Clinical Outcomes in Routine Evaluation (CORE) 10 (Connell \& Barkham, 2007): a 10-item self-report questionnaire of psychological distress. Each item is measured on a 5-point scale ranging from 0 (not at all) to 4 (most or all of the time) with a higher score indicating higher severity.

c) The Mental Health Confidence Scale (MHCS) (Carpinello, Knight, Markowitz, \& Pease, 2000): a 16-item self-report measure of self-efficacy in relation to mental health with domains of optimism, coping and advocacy. Each item is measured on a six point Likert scale that ranges from 1 (very non-confident) to 6 (very confident) with a higher score indicating higher levels of self-efficacy.

Adverse event data were also collected at 6-month follow-up. This included the number of participants who made contact with intensive home treatment teams (IHTT) or accident and 
emergency (A\&E) services (in relation to mental health), the number of deaths related to mental health (e.g. suicide) and the number of participants not discharged at follow-up.

\section{Clusters and participants}

Two wards (i.e. clusters) were recruited, one of which received EDAPTS plus treatment as usual (TAU) while one received just TAU. Wards (i.e. clusters) were not randomised. Individuals admitted to the wards between October 2015 and October 2016 were invited to participate, if they were: (i) aged 16-65, (ii) deemed able to give informed consent by nursing or medical staff; (iii) admitted to one of the acute wards at time of first data collection point; (iv) classed as 'stabilised' by medical staff or nursing staff, and unlikely to find questionnaires too distressing and (v) likely to be admitted for more than 3 days (as judged by ward staff). Participants were excluded if they had received a diagnosis of moderate or severe and profound learning disability, dementia or organic mental disorder, were unable to understand self-report questionnaires due to impaired cognitive processes, concentration, had severe cognitive difficulties which may hinder engagement in talking therapy interventions, did not speak English, presented unsafe behaviour, i.e. severe hostility/aggression or sexually uninhibited behaviour towards staff or were discharged or moved to a different ward within 2 days of completing the first assessment. There was no restriction on mental health diagnosis. Participants were excluded from the study if they moved ward or were discharged within two days of entering the study. Ward staff were consulted to identify patients meeting the eligibility criteria, and written consent from patients was obtained by the researcher (CP). Participants were not randomly allocated to groups due to the cluster design of the study (Eldridge \& Kerry, 2012).

Sample size 
As this was a feasibility study and the main clinical outcome was binary (i.e. proportion of readmissions), the recommended target sample size was 120 (Teare, et al., 2014). To account for $20 \%$ attrition, based on previous studies conducted in this setting (Lewis et al., 2002), the estimated target sample size for this study was 150 .

\section{Control setting}

The control ward was a low security, 25 bed, mixed sex ward for acute inpatients residing in Scotland, and was selected, from a choice of two, because it had no current access to psychological interventions. At the time of recruitment usual care included initial consultation with a psychiatrist on admission, followed by weekly or 'as-required' reviews, formation of a care plan, patient involvement in care planning, occupational therapy input, pharmacotherapy input and assignment of a key worker (staff nurse). No psychological intervention was routinely available or provided.

\section{Intervention Setting}

The intervention ward was a low security 40 bed acute mental health inpatient ward for people residing in Scotland and was divided into two separately secure sections: male and female. At study onset, the ward had three resident consultant psychiatrists, four junior doctors and a continuous flow of medical students. Additionally, two senior charge nurses (one for each section), two charge nurses (one for each section) and a team of nurses and nursing assistants (each section had dedicated staff teams) were employed during data collection. Outside the medical team, the ward also had an Occupational Therapist, a Recreation Officer and access to EDAPTS. EDAPTS was provided specifically for this study, and included a Consultant Clinical Psychologist who provided three hours of individual sessions per week and a Clinical Psychologist/Advance Nurse Practitioner (17.5 hours per week) who was responsible for 
running groups, providing individual sessions for patients, providing clinical supervision to ward staff delivering components of the psychological intervention and facilitating group reflective practice (see below).

\section{E-Acute Psychological Inpatient Therapy Service (EDAPTS)}

The EDAPTS model of intervention was based on the Woodhaven Approach by Clarke and Wilson (2009), more recently known as Comprehend, Cope and Connect (CCC) (Clarke \& Nicholls, 2018), which offers patients individual therapy and CBT- and 'third-wave'-based group therapies. The model is underpinned by a theoretical framework of cognitive process, which conceptualises shared dysfunctional processing and high levels of arousal across diagnoses (see Barnard \& Teasdale, 1991; Clarke, 2009; Teasdale, 1993) for detailed descriptions). EDAPTS' primary aim is to decrease patient distress and increase self-efficacy, through increased access to group and individual therapies. Other aims are to improve therapeutic milieu by developing a psychologically minded workforce. This is achieved by providing psychological training, weekly group reflective practice and the opportunity to facilitate groups therapies and receive associated clinical supervision for staff (Clarke \& Wilson, 2009). Individual sessions involved emotion focused formulation and aimed to identify emotional distress and unhelpful coping mechanisms (I Clarke, 2015). Weekly group therapies were adopted from the Woodhaven Approach (Clarke \& Wilson, 2009). 'Anxiety and Stress Management' provides two sessions of psycho-education relating to the physiological safety system along with mindfulness and arousal management skills. Three 'Making Friends with Yourself' sessions teach self-soothing and self-compassion skills to increase self-esteem. Four 'What is Real and What is Not?' aims to normalise non-shared experiences, identify triggers of unusual experiences and to recognise and reduce arousal. An additional biweekly, 6-session, emotion regulation group, called 'Living Well with Emotions', was also offered to improve 
emotion regulation and distress tolerance. To accommodate the unpredictable nature of acute inpatient services, patients chose the type and number of sessions to attend. Individual sessions were delivered by the clinical psychology team who, with ward staff nurses, also co-facilitated the groups - with the eventual goal for staff nurses to lead these. Staff were offered one-day training to introduce CBT-based skills along with the values and rationale of the model and introduce, two-day training of mentalization-based skills, aiming to improve staff-patient interactions, and weekly group reflective practice and clinical supervision, where necessary, to provide a forum to problem solve.

\section{Analysis}

The revised primary outcomes were reported descriptively (counts, percentages, means and standard deviations). The revised secondary outcomes (i.e., clinical change) were analysed on an intention-to-treat (ITT) basis, where all participants were included, and a per-protocol (PP) basis, where the analysis was limited to participants who received at least one session of psychological therapy. For continuous outcomes, we calculated means, standard deviations (SD) and change in mean scores (SD). Group differences in mean change were estimated using standardised effect sizes (Hedges's g) and 95\% confidence intervals (95\% CI) to indicate the direction of effect and help inform the sample size of a future definitive trial. Hedges's $g$ was interpreted using Cohen's (1988) guidelines: 0.2 signifies a small effect, 0.5 a medium effect and 0.8 a large effect. Count data, percentages and absolute risk differences, with 95\% confidence intervals, are presented for dichotomous outcomes of effect. Missing data was assessed and where deemed to be missing at random (MAR), multiple imputation was used. A sensitivity analysis, involving data reanalysis without multiple imputation, was conducted to check the consistency of result. SPSS 23 (IBM, 2016) was used for all analyses. 


\section{Results}

\section{Sample characteristics}

A total of 96 participants entered the study (see Figure 1). Sixty-three received EDAPTS plus TAU and 33 received TAU alone. Sample characteristics and baseline symptom severity are provided in Table 1 . The sample mean age was $43.38(\mathrm{SD}=11.43)$ and $49 \%$ of participants were male. A high percentage of participants had previous admissions in both groups (59-61\%) and they had received a variety of diagnoses, with psychosis and schizophrenia being most common in both groups (24-46\%). Slightly more participants receiving EDAPTS were diagnosed with psychosis or schizophrenia.

Primary outcomes - feasibility \& trial parameters

\section{Eligibility}

Of those patients who entered the wards, $63 \%$ were eligible to take part. A notable proportion were excluded because they were discharged or moved wards within 2 days of completing baseline measures, were unlikely to be admitted for more than three days or that they were not 'stabilised', as judged by ward staff $(15 \%, 27 \%$ or $20 \%$, respectively) (see Figure 1$)$. Although not directly measured, it was observed that some patients were wrongly excluded, i.e. were admitted for longer than initially anticipated.

\section{Recruitment}

The average number of participants recruited was 4 per month, which was lower than the anticipated rate of 12.5 per month. Recruitment was therefore stopped before reaching the target sample size $(n=150)$. 


\section{Consent}

All wards invited to participate consented. Of the patients deemed eligible to participate in the study, 47\% (N=116) consented. Reasons for not consenting were not formally recorded in this study, however two patients reported feeling 'uncomfortable being part of a study' or that it was 'too much', two patients had concerns that ward staff would think they were ill as a result of completing questionnaires or that they could not concentrate for long enough. A further three patients reported that they were 'not the right person', that their experience was too personal to share or that they felt 'ticking boxes' did not capture their experience.

\section{Randomisation procedures}

Neither participants nor clusters were randomised.

\section{Blinding procedures}

Participant blinding was not possible due to the nature of the intervention. Blinding of assessors and personnel was not possible due to the absence of randomisation and setting of the study.

\section{Implementation and engagement with the intervention}

Of those in the EDAPTS arm, $51 \%(n=32)$ received at least one session of either individual or group psychological intervention. Reasons for non-engagement were not formally recorded, however $10(16 \%)$ participants were unable to receive it due to limited therapy resource or unexpected discharge. Over the study period a total of 133 therapy sessions (group and individual) were delivered to 32 participants, who received a median of 3 sessions overall $(\mathrm{IQR}=4)$. A total of 105 individual sessions ('Emotion focused formulation') were delivered to 16 participants, who received a median of $3.5(\mathrm{IQR}=4)$. These were primarily provided by a 
clinical psychologist and advanced nurse practitioner. Three of the four groups were delivered, and 28 group sessions were delivered overall to 20 participants (median groups received=2, IQR=2). These groups included 15 sessions of 'Living well with emotions', four sessions of 'anxiety and stress management' and 9 sessions of 'Making friends with yourself'. The hearing voices group was not delivered due to limited therapy resource. Nursing staff reported having little time to deliver the groups after completing their usual ward tasks.

\section{Completion of outcome measures}

Data completion rates were $92 \%, 93 \%$ and $53 \%$ for readmission, clinical outcome assessments at post-intervention, and clinical outcome assessment at follow-up (Tables 2). Follow-up retention rates were similar across groups (intervention $=52 \%$; control $=55 \%$ ).

\section{Retention (trial attrition)}

Given the flexible nature of the intervention (i.e. patients were encouraged to attend as few or as many therapy sessions as they chose), treatment retention/attrition could not be recorded. Retention in the trial was indicated by completion rates of questionnaires (see completion of outcome measures).

\section{Summary of all components (decision-making after the feasibility trial)}

Key problems identified against Shanyinde, et al.'s (2011) key methodological features include eligibility criteria, delivery of group therapy sessions, intervention engagement and completion of follow-up questionnaires. See appendices for detail of the full ADePT process (Bugge, et al., 2013).

\section{Secondary outcomes - clinical change}




\section{Readmission}

Where participants had moved country or health board area $(n=5 ; 5 \%)$, died during the followup period $(2 ; 2 \%)$ or had not been discharged from the index admission at 6-month follow-up (1; 1\%), readmission data was unavailable. Both ITT and PP analyses suggested there was little between-group differences in readmission rates and the direction of effect only marginally favoured EDAPTS at follow-up (ITT: EDAPTS 29\%; TAU 33\%; PP: EDAPTS 31\%; TAU $33 \%)$. This corresponds to an absolute risk difference of $-5 \%$ (95\% CI: $-24 \%, 14 \%)$ at postintervention and $-2 \%(95 \% \mathrm{CI}:-24 \%, 0 \%)$ at follow-up.

Psychological distress and mental health related self-efficacy: Post-intervention (Table 3)

The direction of effects derived from ITT analyses favoured the intervention, however most differences were either trivial or small-moderate in magnitude and all 95\% CI overlapped zero. The most promising effects were found for indicators of overall psychological distress (e.g., BSI-18 total score, $\mathrm{SMD}=-0.48, \mathrm{CI}-0.97,0.06$ ), and mental health related advocacy (MHCS advocacy, $\mathrm{SMD}=0.48, \mathrm{CI}-0.02,0.97)$, however again, all 95\% CI overlapped zero.

Psychological distress and mental health related self-efficacy: Follow-up (Table 4)

Both analyses favoured the intervention on most outcomes, with the exception of the BSI-18 total score and the BSI-18 somatization and depression subscales under ITT principles and the BSI-18 depression subscale under PP principles. The magnitude of effects was larger and more promising on most outcomes of mental health related self-efficacy, particularly the advocacy subscale $(\mathrm{SMD}=0.47,-0.02,0.97)$, however all 95\% CI overlapped zero.

\section{Sensitivity analysis}


Using ITT analyses, all follow-up effect size estimates decreased when missing data was imputed however the differences were marginal (Appendix C). A similar pattern emerged for PP analyses, with the exception of BSI-18 depression, CORE-10 problems and CORE-10 total (see Appendix D).

\section{Adverse events}

There were two deaths in TAU and none in EDAPTS. Under ITT analyses, fewer people receiving EDAPTS had contact with $\mathrm{A} \& \mathrm{E}(\mathrm{N}=11 ; 17 \%)$ or the Intensive Home Treatment Team (IHTT) $(\mathrm{N}=8,13 \%)$, compared to TAU (A\&E 27\%; IHTT; 27\%). Similar findings were found for the PP analyses. One person in the trial (EDAPTS arm) had not been discharged from hospital by the time of the follow-up assessments.

\section{Discussion}

\section{Summary}

To our knowledge, this is the first study to examine the feasibility of delivering and evaluating a cross-diagnostic, CBT-based psychological intervention service in acute psychiatric inpatient care. Our preliminary outcome data suggests the EDAPTS intervention showed some promise on outcomes of distress and self-efficacy, but not readmission. Early evidence also suggests it may be associated with a lower rate of emergency or crisis contact following discharge. Whether these pilot data reflect a causal effect of the intervention will remain unclear until a rigorous cluster-randomised controlled trial is conducted. Our results also suggest that readmission data can be gathered reliably, however the lack of clear change on this outcome suggests either refinement of the intervention is required, or that a future trial should focus instead on psychological distress, self-efficacy or post-discharge crisis care. However our trial 
demonstrates the challenges in assessing some of these outcomes reliably at follow-up, whereas the readmission data remained largely complete.

Is it feasible to provide the EDAPTS intervention in the Acute Psychiatric Inpatient Setting?

We have shown that individual therapy sessions can be delivered with relative ease in the acute environment, whereas, in contrast to previous work (Araci \& Clarke, 2016; Durrant, Clarke, Tolland, \& Wilson, 2007), delivery of group interventions was challenging. This disparity may be due to reliance on ward staff nurses or less resource from the psychological team to deliver groups. Although not formally recorded, perceived barriers to nurses routinely delivering groups include staff shortages, staff sickness and inflexible rotas (i.e. rotational night shifts). A busy workload meant these other duties had to be prioritised over therapeutic work. Furthermore, several psychologically-trained ward staff left during the study period.

Similar barriers have been, and continue to be, reported in inpatient psychiatric services (Berry et al., 2016; Clarke \& Wilson, 2009; McCann \& Bowers, 2005). As key aims of nurse led group therapies are to increase access to psychological therapies and to improve therapeutic milieu, through increased psychological knowledge and skills within different staff groups, this has important implications for the reach of the intervention to both service users and staff. Further work is needed to embed staff-run group therapies in routine practice, and this may involve strategies to protect time and ensure managerial support.

Unlike other studies of inpatient psychological intervention (Berry, Haddock, Kellett, Awenat, et al., 2016; Donaghay-Spire, McGowan, Griffiths, \& Barazzone, 2016), we observed that managerial staff and psychiatrists did not attend relevant psychological training. This may 
suggest either a lack of support for the therapeutic approaches, time and resource restrictions, poor intervention promotion or disparity between disciplinary models of mental illness - further research is required to establish the precise reasons. As successful delivery and evaluation of new interventions rely heavily on agreement and commitment from the organisation and staff at all levels (Berry, Haddock, Kellett, Roberts, et al., 2016; Berry \& Haddock, 2008; De Silva et al., 2014; Ince, Haddock, \& Tai, 2016), this issue warrants further investigation, perhaps through the use of qualitative methods.

A large proportion of participants in the intervention group did not engage with the intervention, i.e. did not receive at least one group or individual session. This may indicate that the intervention was unacceptable to patients. However, given the difficulties in group therapy delivery, it is possible that psychological therapy was not available for, or offered to, some participants. The demand for psychological therapies during acute admission, with associated reasons, should be investigated in future research to inform the acceptability and reach of the intervention.

Is it feasible to evaluate the EDAPTS intervention in the Acute Psychiatric Inpatient Setting?

Completion of follow-up questionnaires was low (48\%). Although this is not unusual in a population with severe mental illness (Owens, et al., 2015), it can greatly limit the validity of parameter estimates (Dunn, 2013; Hutton et al., 2012). Therefore, while sensitivity analyses suggested appropriate methods were used to handle missing data, in future additional measures (e.g. increased contact with participants and incentives) should be implemented to reduce attrition and increase internal validity. During the study period, some of the exclusion criteria proved to be problematic. It took several weeks to assess in the best way who was eligible to 
participate and there was a need to tighten up this process. Furthermore, some people may have been excluded who were in fact eligible therefore the numbers approached to participate could have been higher than that presented. This demonstrates the challenges of conducting a trial in a fast paced service with a high patient turnover. To progress to a larger trial it is essential to identify the best methods to recruit a representative sample while maintaining internal validity. This may involve altering the design of the trial or mode of therapy.

Is EDAPTS a promising intervention for acute psychiatric inpatients?

Most estimated treatment effects favoured the intervention to varying degrees, depending on the chosen outcome and analysis. On most outcomes of psychological distress or self-efficacy, group differences tended to be smaller when analysed under ITT, as opposed to PP, principles. This disparity probably reflects the large proportion of non-engaging patients and suggests therapeutic milieu had little effect alone. It is also possible that more direct and sensitive, measures are required to detect change in therapeutic milieu, e.g. outcomes that measure ward atmosphere or therapeutic milieu directly. Minimal group differences were observed on the primary outcome (i.e. number of readmissions) whether analysed under ITT or PP principles. Number of readmissions may lack sensitivity to detect group differences, in which case the planned primary outcome should be altered. Adverse events, for example, may be more suitable. However, it may also be that inconsistent and sparse delivery of group sessions diluted the impact of the intervention (Moore et al., 2015) or that staff referred more chronically ill patients (i.e. revolving door patients) to therapy more readily.

\section{Strengths and limitations}


Our study has several strengths; it builds upon initial exploratory studies by examining the psychological intervention model as a whole, by using a control group, and by measuring readmission and safety (adverse events). However, it also has some limitations. We do not yet know whether it is feasible to randomise at the cluster level (i.e., inpatient wards), and we have yet to examine the feasibility of using rater-blinding in a cluster trial context. Such parameters should be tested before progressing to an adequately powered, single-blind, definitive cluster RCT. It is also recommended that important contextual factors be recorded and accounted for in future trial designs, e.g. by using stratification or a stepped wedge design (Moore et al., 2015).

\section{Clinical implications}

To increase the reach, and outcomes, of the intervention to patients and staff, delivery of nurseled groups may need to be embedded into routine clinical practice. To do so, nurses may need fewer groups to focus on, protected time to deliver groups and attend training and supervision. Additionally, sufficient psychological resource is needed to provide training and supervision. Negotiations with service providers and service managers may be required to agree on time and resource availability.

\section{Research recommendations}

Future trials in this setting are likely to benefit from further development and feasibility work, focused on testing the feasibility of other trial parameters such as randomisation and blinding and modelling the intervention. This may involve using case studies and series, theory of change or logic modelling techniques (Craig et al., 2008). Qualitative investigation of multidisciplinary staff perspectives of the intervention, and of patients perspectives of what a successful 
intervention means, would inform the latter and aid intervention refinement, and trial design. Furthermore, intervention fidelity (i.e., adherence to the intervention by staff) should be properly defined and measured, and further consideration needs to be given to eligibility criteria to ensure an appropriate balance of internal and external validity. Methods to evaluate change in therapeutic milieu and ward atmosphere should also be devised, and further evaluation of strategies to reduce attrition at follow-up may be required. Finally, it may also be useful to measure potential moderators of treatment efficacy, such as staff burnout and degree of psychological knowledge. 


\section{References}

Araci, D., \& Clarke, I. (2016). Investigating the efficacy of a whole team, psychologically informed, acute mental health service approach. Journal of Mental Health, 8237, 1-5. https://doi.org/10.3109/09638237.2016.1139065

Barnard, P. J., \& Teasdale, J. D. (1991). Interacting cognitive subsystems: A systemic approach to cognitive-affective interaction and change. Cognition \& Emotion, 5(1), 139. https://doi.org/10.1080/02699939108411021

Berry, K., \& Haddock, G. (2008). The implementation of the NICE guidelines for schizophrenia: Barriers to the implementation of psychological interventions and recommendations for the future. Psychology and Psychotherapy: Theory, Research and Practice, (81), 419-436. https://doi.org/10.1348/147608308X329540

Berry, K., Haddock, G., Kellett, S., Awenat, Y., Szpak, K., \& Barrowclough, C. (2016). Understanding Outcomes in a Randomized Controlled Trial of a Ward-based Intervention on Psychiatric Inpatient Wards: A Qualitative Analysis of Staff and Patient Experiences. Journal of Clinical Psychology, pp. 1-15. https://doi.org/10.1002/jclp.22434

Berry, K., Haddock, G., Kellett, S., Roberts, C., Drake, R., \& Barrowclough, C. (2016). Feasibility of a ward-based psychological intervention to improve staff and patient relationships in psychiatric rehabilitation settings. British Journal of Clinical Psychology, 55(3), 236-252. https://doi.org/10.1111/bjc.12082

Bickley, H., Isabelle Hunt, B. M., Windfuhr, K., Shaw, J., Appleby, L., \& Kapur, N. (2013). Suicide Within Two Weeks of Discharge From Psychiatric Inpatient Care: A CaseControl Study. Psychiatric Services, 64, 653-659.

https://doi.org/10.1176/appi.ps.201200026

British Psychological Society. (2012). Commissioning and Delivering Clinical Psychology in 
Acute Adult Mental Health Care. Retrieved from http://www.bps.org.uk/system/files/Public files/DCP/cat-1138.pdf

British Psychological Society. (2015). The Commission to Review the Provision of Acute Inpatient Psychiatric Care for Adults in England, Wales and Northern Ireland: background briefing paper. Retrieved from https:/www.bps.org.uk/system/files/userfiles/DCP Psychosis \%2526 Complex Mental Health Faculty/public/bps-response_provision_of_inpatient_psychiatric_care_march_2015.pdf

Brueton, V. C., Tierney, J. F., Stenning, S., Meredith, S., Harding, S., Nazareth, I., \& Rait, G. (2014). Strategies to improve retention in randomised trials: a Cochrane systematic review and meta-analysis. British Medical Journal Open, 4(2), e003821. https://doi.org/10.1136/bmjopen-2013-003821

Bugge, C., Williams, B., Hagen, S., Logan, J., Glazener, C., Pringle, S., \& Sinclair, L. (2013). A process for Decision-making after Pilot and feasibility Trials (ADePT): development following a feasibility study of a complex intervention for pelvic organ prolapse. Trials, 14(1), 353. https://doi.org/10.1186/1745-6215-14-353

Campbell, M., Fitzpatrick, R., Haines, A., Kinmonth, A. L., Sandercock, P., Spiegelhalter, D., \& Tyrer, P. (2000). Framework for design and evaluation of complex interventions to improve health Framework for trials of complex interventions. British Medical Journal, 321(7262), 694-6. https://doi.org/10.1136/bmj.321.7262.694

Care Quality Commission. (2015). Right here, right now - help, care and support during a mental health crisis (summary). Care Quality Commission. https://doi.org/10.1016/j.ogrm.2012.05.002

Carey, T. A., Tai, S. J., Mansell, W., Huddy, V., Griffiths, R., \& Marken, R. S. (2017). Improving professional psychological practice through an increased repertoire of research methodologies: Illustrated by the development of MOL. Professional 
Psychology: Research and Practice, 48(3), 175-182. https://doi.org/10.1037/pro0000132

Carpinello, S. E., Knight, E. L., Markowitz, F. E., \& Pease, E. A. (2000). The development of the Mental Health Confidence Scale: A measure of self-efficacy in individuals diagnosed with mental disorders. Psychiatric Rehabilitation Journal, 23(3), 236-243.

https://doi.org/10.1037/h0095162

Clarke, I. (2009). Prioneering a cross-diagnostic approach founded in cognitive science. In I. Clarke \& H. Wilson (Eds.), Cognitive behaviour therapy for acute inpatient mental health units: Working with clients, staff and the milieu (1st ed., pp. 65-76). New York, NY: Routledge.

Clarke, I. (2015). The emotion focused formulation approach: Bridging individual and team formulation. Clinical Psychology Foum, 275, 28-33. Retrieved from http://www.isabelclarke.org/clinical/icsthird.shtml

Clarke, I., \& Nicholls, H. (2018). Third wave CBT integration for individuals and teams : comprehend, cope, and connect. Routledge. Retrieved from https://www.routledge.com/Third-Wave-CBT-Integration-for-Individuals-and-TeamsComprehend-Cope/Clarke-Nicholls/p/book/9781138226906

Clarke, I., \& Wilson, H. (Eds.). (2009). Cognitive behaviour therapy for acute inpatient mental health units: Working with clients, staff and the milieu. London, UK: Routledge Forthcoming. Retrieved from http://ovidsp.ovid.com/ovidweb.cgi?T=JS\&PAGE=reference $\& D=$ psyc6\&NEWS $=$ N\&A $\mathrm{N}=2008-07840-000$

Cohen, J. (1988). Statistical power analysis for the behavioral sciences (1st ed.). Hillsdale, NJ: Erlbaum Associates.

Connell, J., \& Barkham, M. (2007). Core-10 User Manual: Version 1.1. CORE System Trust \& CORE Information Management SYstems Ltd. Retrieved from 
http://www.mhtu.co.uk/outcome-monitoring/outcome-core-10-manual.pdf

Craig, P. (2012). Developing and evaluating complex interventions. Retrieved from www.mrc.ac.uk/complexinterventionsguidance

Craig, P., Dieppe, P., Macintyre, S., Michie, S., Nazareth, I., \& Petticrew, M. (2013).

Developing and evaluating complex interventions: The new Medical Research Council guidance. International Journal of Nursing Studies, 50(5), 585-592. https://doi.org/10.1016/j.ijnurstu.2012.09.010

Craig, P., Dieppe, P., Macintyre, S., Michie, S., Nazareth, I., Petticrew, M., \& Medical Research Council Guidance. (2008). Developing and evaluating complex interventions: the new Medical Research Council guidance. British Medical Journal (Clinical Research Ed.), 337(7262), a1655. https://doi.org/10.1136/bmj.a1655

De Silva, M. J., Breuer, E., Lee, L., Asher, L., Chowdhary, N., Lund, C., \& Patel, V. (2014). Theory of Change: A theory-driven approach to enhance the Medical Research Council's framework for complex interventions. Trials, 15(1). https://doi.org/10.1186/1745-6215$15-267$

Derogatis, L. (2001). Brief Symptom Inventory (BSI)-18. Administration, scoring and procedures manual. Minneapolis: NCS Pearson.

Dodds, P., \& Bowles, N. (2001). Psychiatric nursing and organizational power: rescuing the hidden dynamic. Journal of Psychiatric and Mental Health Nursing, 8(2), 173-7. Retrieved from http://www.ncbi.nlm.nih.gov/pubmed/11882123

Donaghay-Spire, E. G., McGowan, J., Griffiths, K., \& Barazzone, N. (2016). Exploring narratives of psychological input in the acute inpatient setting. Psychology and Psychotherapy: Theory, Research and Practice, 89(4), 464-482.

https://doi.org/10.1111/papt.12081

Dunn, G. (2013). Pragmatic trials of complex psychosocial interventions: methodological 
challenges. Epidemiology and Psychiatric Sciences, 22(02), 105-109.

https://doi.org/doi:10.1017/S2045796013000048

Durrant, C., Clarke, I., Tolland, A., \& Wilson, H. (2007). Designing a CBT service for an acute inpatient setting: A pilot evaluation study. Clinical Psychology and Psychotherapy, 14(2), 117-125. https://doi.org/10.1002/cpp.516

Eldridge, S., \& Kerry, S. M. (2012). A practical guide to cluster randomised trials in health services research. John Wiley \& Sons.

Eldridge, S. M., Chan, C. L., Campbell, M. J., Bond, C. M., Hopewell, S., Thabane, L., \& Lancaster, G. A. (2016). CONSORT 2010 statement: extension to randomised pilot and feasibility trials. Pilot and Feasibility Studies, 2(1), 64. https://doi.org/10.1186/s40814$016-0105-8$

Eldridge, S. M., Lancaster, G. A., Campbell, M. J., Thabane, L., Hopewell, S., Coleman, C. L., \& Bond, C. M. (2016). Defining feasibility and pilot studies in preparation for randomised controlled trials: Development of a conceptual framework. PLoS ONE, 11(3), 1-22. https://doi.org/10.1371/journal.pone.0150205

Gordon, W. (2005). Launching the Tidal Model: evaluating the evidence. Journal of Psychiatric and Mental Health Nursing, 12(12), 703-712. Retrieved from http://www.bills-christiantrek.me.uk/Christiantrek/Mental_Health_files/LAUNCHING THE TIDAL MODEL.pdf

Higgins, J., \& Green, S. (2011). Cochrane Handbook for Systematic Reviews of Interventions | Cochrane Training. The Cochrane Collaboration. Retrieved from http://training.cochrane.org/handbook

Hill, G., Clarke, I., \& Wilson, H. (2009). The "Making Friends with Yourself" and the "What is Real and What is Not" groups. In I. Clarke \& H. Wilson (Eds.), Cognitive Behaviour Therapy for Acute Inpatient Mental Health Units: Working with Clients, Staff and the 
Milieu (1st ed., pp. 161-172). New York, NY: Routledge.

Hutton, P., Morrison, A. P., Yung, A. R., Taylor, P. J., French, P., \& Dunn, G. (2012). Effects of drop-out on efficacy estimates in five Cochrane reviews of popular antipsychotics for schizophrenia. Acta Psychiatrica Scandinavica, 126(1), 1-11.

https://doi.org/10.1111/j.1600-0447.2012.01858.x

IBM. (2016). IBM SPSS - IBM Analytics. Retrieved from http://www.ibm.com/analytics/us/en/technology/spss/

Ince, P., Haddock, G., \& Tai, S. (2016). A systematic review of the implementation of recommended psychological interventions for schizophrenia: Rates, barriers, and improvement strategies. Psychology and Psychotherapy: Theory, Research and Practice, 89(3), 324-350. https://doi.org/10.1111/papt.12084

Information Services Division Scotland. (2012). Mental Health (Psychiatric) Hospital Activity Statistics year ending 31 March 2012. Retrieved from http://www.isdscotland.org/Health-Topics/Mental-Health/Publications/2012-12-18/201212-18-MentalHealth-Report.pdf?99358767272

Jacobsen, P., Morris, E., Johns, L., \& Hodkinson, K. (2011). Mindfulness Groups for Psychosis; Key Issues for Implementation on an Inpatient Unit. Behavioural and Cognitive Psychotherapy, 39, 349-353. https://doi.org/10.1017/S1352465810000639 Jacobsen, P., Peters, E., \& Chadwick, P. (2016). Mindfulness-Based Crisis Interventions for patients with psychotic symptoms on acute psychiatric wards (amBITION study): protocol for a feasibility randomised controlled trial. Pilot and Feasibility Studies, 2(1), 43. https://doi.org/10.1186/s40814-016-0082-y

Janner, M. (2007). From the inside out: Star Wards - lessons from within acute in-patient wards. Journal of Psychiatric Intensive Care, 3(2), 75-78. https://doi.org/http://dx.doi.org/10.1017/S1742646407001136 
Jauhar, S., McKenna, P. J., Radua, J., Fung, E., Salvador, R., \& Laws, K. R. (2014). Cognitive-behavioural therapy for the symptoms of schizophrenia: Systematic review and meta-analysis with examination of potential bias. British Journal of Psychiatry, 204(1), 20-29. https://doi.org/10.1192/bjp.bp.112.116285

Kleschinsky, J. H., Bosworth, L. B., Nelson, S. E., Walsh, E. K., \& Shaffer, H. J. (2009). Persistence Pays Off: Follow-Up Methods for Diffi cult-to- Track Longitudinal Samples*. Alcohol Drugs, 70, 751-761. https://doi.org/10.15288/jsad.2009.70.751

Lancaster, G. A. (2015). Pilot and feasibility studies come of age! Pilot and Feasibility Studies, 1(1), 1. https://doi.org/10.1186/2055-5784-1-1

Lewis, S., Tarrier, N., Haddock, G., Bentall, R., Kinderman, P., Kingdon, D., ... Dunn, G. (2002). Randomised controlled trial of cognitive -- behavioural therapy in early schizophrenia : acute-phase outcomes Randomised controlled trial of cognitive behavioural therapy in early schizophrenia : British Journal of Psychiatry, 181(43), 9197. https://doi.org/10.1192/bjp.181.43.s91

McCann, E., \& Bowers, L. (2005). Training in cognitive behavioural interventions on acute psychiatric inpatient wards. Journal of Psychiatric and Mental Health Nursing, 12(2), 215-222. https://doi.org/10.1111/j.1365-2850.2004.00822.x

Mind. (2004). Ward watch: Mind's campaign to improve hospital conditions for mental health patients. Retrieved from http://news.bbc.co.uk/nol/shared/bsp/hi/pdfs/07_09_04_mindsummary.pdf

Moore, G. F., Audrey, S., Barker, M., Bond, L., Bonell, C., Hardeman, W., ... Baird, J. (2015). Process evaluation of complex interventions: Medical Research Council guidance. BMJ, 350(6). https://doi.org/10.1136/bmj.h1258

Owen, M., Sellwood, W., Kan, S., Murray, J., \& Sarsam, M. (2015). Group CBT for psychosis: A longitudinal, controlled trial with inpatients. Behaviour Research and 
Therapy, 65, 76-85. https://doi.org/10.1016/j.brat.2014.12.008

Royal College of Physicians. (2007). Guidelines on the practice of ethics committees in medical research with human participants Fourth edition. Retrieved from https://cdn.shopify.com/s/files/1/0924/4392/files/guidelines-practice-ethics-committeesmedical-research.pdf?11143599859970562352=

Royal College of Psychiatrists. (2010). Accreditation for Inpatient Mental Health Services (AIMS) Standards for Inpatient Wards-Working-Age Adults. Retrieved from https://www.rcpsych.ac.uk/pdf/Standards for Inpatient Wards - Working Age Adults Fourth Edition.pdf

Sainsbury Centre for Mental Health. (2005). Acute Care 2004: A National Survery of Adult Psychiatric Wards in England. National Institute for Mental Health in England Acute Inpatient Care Programme. Retrieved from https://www.researchgate.net/publication/242558279_Acute_Care_2004_A_national_sur vey_of_adult_psychiatric_wards_in_England

Schizophrenia Commission. (2012). The Abandoned Illness: a Report from the Schizophrenia Commission. Retrieved from https://www.rethink.org/media/514093/TSC_main_report_14_nov.pdf

Shanyinde, M., Pickering, R. M., \& Weatherall, M. (2011). Questions asked and answered in pilot and feasibility randomized controlled trials. BMC Medical Research Methodology, 11(117). https://doi.org/10.1186/1471-2288-11-117

Shaw, E. K., Howard, J., West, D. R., Crabtree, B. F., Nease, D. E., Tutt, B., \& Nutting, P. A. (2012). The Role of the Champion in Primary Care Change Efforts. The Journal of the American Board of Family Medicine, 25(5), 676-685.

https://doi.org/10.3122/jabfm.2012.05.110281

Stevenson, C., Barker, P., \& Fletcher, E. (2002). Judgement days: Developing an evaluation 
for an innovative nursing model. Journal of Psychiatric and Mental Health Nursing, 9(3), 271-276. https://doi.org/10.1046/j.1365-2850.2002.00472.x

Teasdale, J. D. (1993). Emotion and two kinds of meaning: Cognitive therapy and applied cognitive science. Behaviour Research and Therapy, 31(4), 339-354. https://doi.org/10.1016/0005-7967(93)90092-9

Turner, D. T., Van Der Gaag, M., Karyotaki, E., \& Cuijpers, P. (2014). Psychological interventions for psychosis: A meta-analysis of comparative outcome studies. American Journal of Psychiatry, 171(5), 523-538. https://doi.org/10.1176/appi.ajp.2013.13081159

Veltro, F., Falloon, I., Vendittelli, N., Oricchio, I., Scinto, A., Gigantesco, A., \& Morosini, P. (2006). Effectiveness of cognitive-behavioural group therapy for inpatients. Clinical Practice and Epidemiology in Mental Health, 2(16). https://doi.org/10.1186/1745-0179$2-16$

Wykes, T., Steel, C., Everitt, B., \& Tarrier, N. (2008). Cognitive behavior therapy for schizophrenia: Effect sizes, clinical models, and methodological rigor. Schizophrenia Bulletin, 34(3), 523-537. https://doi.org/10.1093/schbul/sbm114 
Tables and figures for manuscript

\begin{tabular}{|c|c|c|c|c|c|c|}
\hline \multicolumn{7}{|c|}{$\begin{array}{l}\text { Table } 1 \\
\text { Summary of sample characteristics } \\
\end{array}$} \\
\hline Characteristic & & Intervention (ITT) $(n=63)$ & Control $^{1}(n=33)$ & $\begin{array}{c}\text { Total } \\
(\mathrm{n}=96)\end{array}$ & $\begin{array}{c}\text { Intervention (PP) } \\
(\mathrm{n}=32)^{2}\end{array}$ & $\begin{array}{l}\text { Intervention non- } \\
\text { engagers }(n=31)^{2}\end{array}$ \\
\hline $\begin{array}{l}\text { Baseline } \\
\text { CORE-10 } \\
(\mathrm{M}, \mathrm{SD})\end{array}$ & Total score & $21.63(10.44)$ & $22.27(10.56)$ & $21.85(10.43)$ & $22.72(9.65)$ & $20.52(11.25)$ \\
\hline \multirow{4}{*}{$\begin{array}{l}\text { Baseline BSI } \\
(\mathrm{M}, \mathrm{SD})\end{array}$} & Total & $34.56(20.69)^{3}$ & $37.88(19.76)$ & $35.37(20.30)^{4}$ & $37.06(20.39)^{5}$ & $32.06(21.01)$ \\
\hline & Somatization & $8.76(6.94)^{3}$ & $9.33(6.70)$ & $8.96(6.83)^{4}$ & $10.03(7.45)^{5}$ & $7.48(6.24)$ \\
\hline & Depression & $12.61(8.17)^{3}$ & $13.94(8.48)$ & $13.07(8.26)^{4}$ & $13.29(8.06)^{5}$ & $11.94(8.35)$ \\
\hline & Anxiety & $13.21(8.28)^{3}$ & $13.88(6.97)$ & $13.44(7.82)^{4}$ & $13.84(7.83)^{5}$ & $12.58(8.80)$ \\
\hline \multirow{4}{*}{$\begin{array}{l}\text { MHCS } \\
(\mathrm{M}, \mathrm{SD})\end{array}$} & Total & $54.63(23.60)$ & $58.94(24.82)$ & $56.11(23.98)$ & $52.84(22.06)$ & $56.48(25.32)$ \\
\hline & Optimism & $21.41(9.23)$ & $23.06(9.63)$ & $21.98(9.35)$ & $21.78(8.35)$ & $21.03(10.18)$ \\
\hline & Coping & $22.16(10.94)$ & 22.15 (11.69) & $22.16(11.14)$ & $22.09(10.46)$ & $22.23(11.59)$ \\
\hline & Advocacy & $12.14(4.83)$ & $13.09(4.72)$ & $12.47(4.79)$ & $11.16(4.68)$ & $13.16(4.85)$ \\
\hline Gender (n (\%)) & Male & $35(56 \%)$ & $12(36 \%)$ & $47(49 \%)$ & $17(53 \%)$ & $18(58 \%)$ \\
\hline
\end{tabular}




\begin{tabular}{|c|c|c|c|c|c|c|}
\hline & Female & $28(44 \%)$ & $21(64 \%)$ & $49(51 \%)$ & $15(47 \%)$ & $13(42 \%)$ \\
\hline & Total & $63(100 \%)$ & $33(100 \%)$ & $96(100 \%)$ & $32(100 \%)$ & $31(100 \%)$ \\
\hline \multirow[t]{6}{*}{$\begin{array}{l}\text { Diagnosis } \\
(\mathrm{n}(\%))\end{array}$} & $\begin{array}{l}\text { Bipolar } \\
\text { (manic) }\end{array}$ & $13(21 \%)$ & $10(30 \%)$ & $23(24 \%)$ & $6(19 \%)$ & $7(23 \%)$ \\
\hline & $\begin{array}{l}\text { Bipolar } \\
\text { (depression) }\end{array}$ & $4(6 \%)$ & $2(6 \%)$ & $6(6 \%)$ & $2(6 \%)$ & $2(6 \%)$ \\
\hline & $\begin{array}{l}\text { Personality } \\
\text { disorder }\end{array}$ & $6(10 \%)$ & $5(15 \%)$ & $11(12 \%)$ & $2(6 \%)$ & $4(13 \%)$ \\
\hline & Depression & $11(18 \%)$ & $8(24 \%)$ & $19(20 \%)$ & $6(19 \%)$ & $5(16 \%)$ \\
\hline & $\begin{array}{l}\text { Schizophrenia } \\
\text { and psychosis }\end{array}$ & $29(46 \%)$ & $8(24 \%)$ & $37(39 \%)$ & $16(50 \%)$ & $13(42 \%)$ \\
\hline & Total & $63100 \%$ & $33(100 \%)$ & $96(100 \%)$ & $32(100 \%)$ & $31(100 \%)$ \\
\hline \multicolumn{2}{|c|}{ Age (M, SD) } & $43.03(12.34)$ & $44.03(9.59)$ & $43.38(11.43)$ & $40.75(13.17)$ & $45.38(11.15)$ \\
\hline \multicolumn{2}{|c|}{ Previous admission (n, \%) } & $37(59 \%)$ & $20(61 \%)$ & $57(59 \%)$ & $17(53 \%)$ & $20(65 \%)$ \\
\hline \multicolumn{2}{|c|}{ LOS (median, IQR) } & $34.50(48.75)$ & $24.00(29.50)$ & $28.00(44.00)$ & $35.00(63.00)$ & $33.00(38.00)$ \\
\hline
\end{tabular}

Brief Symptom Inventory Scale (BSI); Clinical Outcomes Routine Evaluation (CORE); Mental Health Confidence Scale (MHCS); Modified intention to treat (ITT); Per protocol(PP); Standard deviation (SD).

1. As all participants in the control group received treatment, the control group includes the same participants whether ITT ot PP. For ease, this group will only be referred to as control group.

2. As subgroup of intervention (ITT).

3. $\mathrm{N}=62$ (one participant chose not to complete the BSI-18 due to similarities with the CORE-10).

4. $\mathrm{N}=95($ see 3$)$.

5. $\mathrm{N}=31$ (see 3$)$.

6. Lower score indicates higher severity. 


\section{Table 2}

Completion rate of each outcome measure at each time point (n (\%))

\begin{tabular}{|c|c|c|c|c|c|c|c|c|c|c|}
\hline \multirow[b]{2}{*}{ Group } & \multicolumn{3}{|c|}{ Pre-intervention } & \multicolumn{3}{|c|}{ Post-intervention } & \multicolumn{3}{|c|}{ 6-month follow-up } & \multirow{2}{*}{$\begin{array}{c}\text { Readmissions } \\
\text { (dichotomous and } \\
\text { continuous) }\end{array}$} \\
\hline & $\begin{array}{c}\text { CORE- } \\
10\end{array}$ & BSI-18 & MHCS & $\begin{array}{c}\text { CORE- } \\
10\end{array}$ & BSI-18 & MHCS & $\begin{array}{c}\text { CORE- } \\
10\end{array}$ & BSI-18 & MHCS & \\
\hline $\begin{array}{l}\text { Intervention } \\
(\mathrm{n}=63)\end{array}$ & $\begin{array}{c}63 \\
(100 \%)\end{array}$ & $\begin{array}{c}62 \\
(98 \%)\end{array}$ & $\begin{array}{c}63 \\
(100 \%)\end{array}$ & $\begin{array}{c}57 \\
(90 \%)\end{array}$ & $\begin{array}{c}56 \\
(89 \%)\end{array}$ & $\begin{array}{c}57 \\
(90 \%)\end{array}$ & $\begin{array}{c}33 \\
(52 \%)\end{array}$ & $\begin{array}{c}32 \\
(51 \%)\end{array}$ & $\begin{array}{c}33 \\
(52 \%)\end{array}$ & $\begin{array}{c}58 \\
(92 \%)\end{array}$ \\
\hline Control $(n=33)$ & $\begin{array}{c}33 \\
(100 \%)\end{array}$ & $\begin{array}{c}33 \\
(100 \%)\end{array}$ & $\begin{array}{c}33 \\
(100 \%)\end{array}$ & $\begin{array}{c}32 \\
(97 \%)\end{array}$ & $\begin{array}{c}32 \\
(97 \%)\end{array}$ & $\begin{array}{c}32 \\
(97 \%)\end{array}$ & $\begin{array}{c}18 \\
(55 \%)\end{array}$ & $\begin{array}{c}18 \\
(55 \%)\end{array}$ & $\begin{array}{c}18 \\
(55 \%)\end{array}$ & $\begin{array}{c}30 \\
(91 \%)\end{array}$ \\
\hline Total $(n=96)$ & $\begin{array}{c}96 \\
(100 \%)\end{array}$ & $\begin{array}{c}95 \\
(99 \%)\end{array}$ & $\begin{array}{c}96 \\
(100 \%)\end{array}$ & $\begin{array}{c}89 \\
(93 \%)\end{array}$ & $\begin{array}{c}88 \\
(92 \%)\end{array}$ & $\begin{array}{c}89 \\
(93 \%)\end{array}$ & $\begin{array}{c}51 \\
(53 \%)\end{array}$ & $\begin{array}{c}50 \\
(52 \%)\end{array}$ & $\begin{array}{c}51 \\
(53 \%)\end{array}$ & $\begin{array}{c}88 \\
(92 \%)\end{array}$ \\
\hline
\end{tabular}


Pre- to post-intervention mean change (ITT analysis)

Pre- to post-intervention mean change (PP analysis)

\begin{tabular}{|c|c|c|c|c|c|c|c|c|c|c|c|c|c|}
\hline Outcome & Group & $\mathbf{n}$ & $\begin{array}{c}\text { Mean } \\
\text { change }\end{array}$ & SD & $\begin{array}{c}g \\
(95 \% \mathrm{CI})\end{array}$ & p & Effect summary $^{1}$ & $\mathbf{n}$ & $\begin{array}{c}\text { Mean } \\
\text { change }\end{array}$ & SD & $\begin{array}{c}g \\
(95 \% \mathrm{CI})\end{array}$ & p & $\begin{array}{c}\text { Effect } \\
\text { summary }{ }^{1}\end{array}$ \\
\hline \multirow{2}{*}{$\begin{array}{l}\text { CORE-10 } \\
\text { Total }\end{array}$} & Intervention & 63 & -7.30 & 9.52 & -0.17 & \multirow{2}{*}{0.45} & \multirow{2}{*}{ Very small } & 32 & -10.15 & 9.84 & \multirow{2}{*}{$\begin{array}{c}-0.48 \\
(-0.97,0.01)\end{array}$} & \multirow{2}{*}{0.06} & \multirow{2}{*}{ Medium } \\
\hline & Control & 33 & -5.78 & 8.16 & $(-0.59,0.26)$ & & & 33 & -5.78 & 8.16 & & & \\
\hline \multirow{2}{*}{ BSI-18 Total } & Intervention & 63 & -12.80 & 18.81 & -0.14 & \multirow{2}{*}{0.51} & \multirow{2}{*}{ Very small } & 32 & -18.24 & 18.10 & \multirow{2}{*}{$\begin{array}{c}-0.48 \\
(-0.97,0.02) \\
\end{array}$} & \multirow{2}{*}{0.05} & \multirow{2}{*}{ Medium } \\
\hline & Control & 33 & -10.31 & 14.71 & $(-0.56,0.28)$ & & & 33 & -10.31 & 14.71 & & & \\
\hline \multirow{2}{*}{$\begin{array}{l}\text { BSI-18 } \\
\text { Somatization }\end{array}$} & Intervention & 63 & -3.72 & 7.06 & -0.13 & \multirow{2}{*}{0.55} & \multirow{2}{*}{ Very small } & 32 & -5.80 & 6.84 & \multirow{2}{*}{$\begin{array}{c}-0.47 \\
(-0.97,0.02) \\
\end{array}$} & \multirow{2}{*}{0.06} & \multirow{2}{*}{ Medium } \\
\hline & Control & 33 & -2.87 & 5.34 & $(-0.55,0.29)$ & & & 33 & -2.87 & 5.34 & & & \\
\hline \multirow{2}{*}{$\begin{array}{l}\text { BSI-18 } \\
\text { Depression }\end{array}$} & Intervention & 63 & -4.27 & 6.75 & -0.01 & \multirow{2}{*}{0.98} & \multirow{2}{*}{ No effect } & 32 & -6.39 & 6.68 & \multirow{2}{*}{$\begin{array}{c}-0.34 \\
(-0.83,0.15) \\
\end{array}$} & \multirow{2}{*}{0.17} & \multirow{2}{*}{$\begin{array}{l}\text { Small to } \\
\text { medium }\end{array}$} \\
\hline & Control & 33 & -4.18 & 6.03 & $(-0.43,0.41)$ & & & 33 & -4.18 & 6.03 & & & \\
\hline \multirow{2}{*}{$\begin{array}{l}\text { BSI-18 } \\
\text { Anxiety }\end{array}$} & Intervention & 63 & -4.81 & 7.06 & -0.23 & \multirow{2}{*}{0.27} & \multirow{2}{*}{ Small } & 32 & -6.05 & 7.01 & \multirow{2}{*}{$\begin{array}{c}-0.42 \\
(-0.91,0.07)\end{array}$} & \multirow{2}{*}{0.08} & \multirow{2}{*}{$\begin{array}{l}\text { Small to } \\
\text { medium }\end{array}$} \\
\hline & Control & 33 & -3.22 & 6.32 & $(-0.65,0.19)$ & & & 33 & -3.22 & 6.32 & & & \\
\hline \multirow{2}{*}{ MHCS Total } & Intervention & 63 & 9.09 & 17.07 & $0.28^{2}$ & \multirow{2}{*}{0.38} & \multirow{2}{*}{ Small } & 32 & 10.44 & 24.49 & $0.29^{2}$ & & \\
\hline & Control & 33 & 4.66 & 13.44 & $(-0.15,0.70)$ & & & 33 & 4.66 & 13.44 & $(-0.20,0.78)$ & 0.43 & Small \\
\hline MHCS & Intervention & 63 & 3.28 & 7.14 & $0.17^{2}$ & 042 & & 32 & 3.98 & 7.30 & $0.29^{2}$ & & \\
\hline Optimism & Control & 33 & 2.18 & 4.83 & $(-0.25,0.59)$ & 0.42 & Very small & 33 & 2.18 & 4.83 & $(-0.20,0.78)$ & 0.23 & Small \\
\hline MHCS & Intervention & 63 & 4.00 & 9.13 & $0.20^{2}$ & & Small & 32 & 4.19 & 16.74 & $0.15^{2}$ & & Verv small \\
\hline Coping & Control & 33 & 2.26 & 6.95 & $(-0.22,0.63)$ & 0.12 & small & 33 & 2.27 & 6.95 & $(-0.34,0.64)$ & 0.99 & very small \\
\hline MHCS & Intervention & 63 & 1.80 & 4.44 & $0.37^{2}$ & & & 32 & 2.27 & 4.70 & $0.48^{2}$ & & \\
\hline Advocacy & Control & 33 & 0.22 & 3.79 & $(-0.05,0.79)$ & 0.09 & small to medium & 33 & 0.22 & 3.79 & $(-0.02,0.97)$ & 0.00 & $\mathrm{~m}$ \\
\hline
\end{tabular}

$\begin{array}{lllllll}\text { Advocacy } & \text { Control } & 33 & 0.22 & 3.79 & (-0.05,0.79) & 0.22\end{array}$ protocol (PP); Standard deviation (SD).

1. Summary is based on Cohen's (1988) benchmarks.

2. Positive effect favours intervention. 
Psychological intervention for acute inpatients: A feasibility trial Table 4 Summary of pre-intervention to follow-up change scores as per ITT and PP analyses

Pre-intervention to follow-up mean change (ITT analysis)

\begin{tabular}{|c|c|c|c|c|c|c|c|c|c|c|c|c|c|}
\hline Outcome & Group & n & $\begin{array}{c}\text { Mean } \\
\text { change }\end{array}$ & SD & $\stackrel{g}{(95 \% ~ C I)}$ & $\mathbf{p}$ & Effect summary $^{1}$ & $\mathbf{n}$ & $\begin{array}{c}\text { Mean } \\
\text { change }\end{array}$ & SD & $\begin{array}{c}g \\
(95 \% \text { CI })\end{array}$ & $\mathbf{p}$ & Effect summary ${ }^{1}$ \\
\hline \multirow{2}{*}{$\begin{array}{l}\text { CORE-10 } \\
\text { Total }\end{array}$} & Intervention & 63 & -6.38 & 11.35 & -0.14 & \multirow{2}{*}{0.51} & \multirow[b]{2}{*}{ Very small effect } & 32 & -8.15 & 11.37 & \multirow{2}{*}{$\begin{array}{c}-0.30 \\
(-0.79,0.19) \\
\end{array}$} & \multirow{2}{*}{0.23} & \multirow{2}{*}{ Small to medium } \\
\hline & Control & 33 & -4.74 & 11.37 & $(-0.56,0.28)$ & & & 33 & -4.74 & 11.37 & & & \\
\hline \multirow{2}{*}{ BSI-18 Total } & Intervention & 63 & -10.53 & 21.75 & 0.02 & \multirow{2}{*}{0.89} & \multirow{2}{*}{ No effect } & 32 & -12.76 & 22.74 & \multirow{2}{*}{$\begin{array}{c}-0.07 \\
(-0.56,0.41)\end{array}$} & \multirow{2}{*}{0.77} & \multirow{2}{*}{ Very small } \\
\hline & Control & 33 & -11.00 & 20.68 & $(-0.40,0.44)$ & & & 33 & -11.00 & 20.68 & & & \\
\hline \multirow{2}{*}{$\begin{array}{l}\text { BSI-18 } \\
\text { Somatization }\end{array}$} & Intervention & 63 & -3.84 & 7.62 & 0.04 & \multirow{2}{*}{0.87} & \multirow{2}{*}{ No effect } & 32 & -4.88 & 7.92 & \multirow{2}{*}{$\begin{array}{c}-0.10 \\
(-0.59,0.39)\end{array}$} & \multirow{2}{*}{0.64} & \multirow{2}{*}{ Very small } \\
\hline & Control & 33 & -4.13 & 6.72 & $(-0.38,0.47)$ & & & 33 & -4.13 & 6.72 & & & \\
\hline \multirow{2}{*}{$\begin{array}{l}\text { BSI-18 } \\
\text { Depression }\end{array}$} & Intervention & 63 & -2.65 & 8.97 & 0.11 & \multirow{2}{*}{0.55} & \multirow{2}{*}{ Very small } & 32 & -3.40 & 9.90 & \multirow{2}{*}{$\begin{array}{c}0.03 \\
(-0.45,0.52) \\
\end{array}$} & \multirow{2}{*}{0.84} & \multirow{2}{*}{ No effect } \\
\hline & Control & 33 & -3.74 & 10.40 & $(-0.31,0.54)$ & & & 33 & -3.74 & 10.40 & & & \\
\hline \multirow{2}{*}{ BSI-18 Anxiety } & Intervention & 63 & -4.09 & 8.73 & -0.11 & \multirow{2}{*}{0.62} & \multirow{2}{*}{ Very small } & 32 & -4.39 & 8.77 & -0.15 & \multirow{2}{*}{0.53} & \multirow{2}{*}{ Very small } \\
\hline & Control & 33 & -3.17 & 7.35 & $(-0.53,0.31)$ & & & 33 & -3.17 & 7.35 & $(-0.64,0.34)$ & & \\
\hline \multirow{2}{*}{ MHCS Total $^{2}$} & Intervention & 63 & 4.05 & 23.34 & 0.35 & \multirow{2}{*}{0.18} & \multirow{2}{*}{ Small to medium } & 32 & 5.80 & 24.38 & 0.40 & \multirow{2}{*}{0.23} & \\
\hline & Control & 33 & -4.70 & 27.57 & $(-0.08,0.77)$ & & & 33 & -4.70 & 27.57 & $(-0.09,0.89)$ & & dium \\
\hline MHCS & Intervention & 63 & 0.98 & 9.68 & 0.37 & & $\mathrm{~S}$ & 32 & 1.54 & 9.90 & 0.41 & 0 & ium \\
\hline Optimism² $^{2}$ & Control & 33 & -2.89 & 11.43 & $(-0.05,0.80)$ & 0 & ait & 33 & -2.89 & 11.43 & $(-0.08,0.90)$ & 0.09 & IIIII \\
\hline MHCS & Intervention & 63 & 2.00 & 11.51 & 0.21 & 059 & Small & 32 & 0.65 & 18.27 & 0.20 & 076 & Small effect \\
\hline Coping $^{2}$ & Control & 33 & -0.66 & 13.73 & $(-0.21,0.64)$ & 0.59 & small & 33 & -0.66 & 13.73 & $(-0.29,0.69)$ & 0.10 & smant enect \\
\hline MHCS & Intervention & 63 & 1.07 & 6.11 & 0.38 & 008 & $\mathrm{~S}$ & 32 & 1.65 & 6.45 & 0.47 & & \\
\hline Advocacy $^{2}$ & Control & 33 & -1.14 & 5.17 & $(-0.05,0.80)$ & 0.08 & small to medium & 33 & -1.14 & 5.17 & $(-0.02,0.97)$ & 0.00 & Medium \\
\hline
\end{tabular}

Brief Symptom Inventory Scale (BSI); Clinical Outcomes Routine Evaluation (CORE); Mental Health Confidence Scale (MHCS); Modified intention to treat (ITT); Per protocol (PP); Standard deviation (SD).

1. Summary is based on Cohen's (1988) benchmarks

2. Positive effect favours intervention. 
Violating inclusion/exclusion criteria ( $\mathrm{n}=116)$ : not 'stabilised' $(\mathrm{n}=27)$, unable to complete questionnaire $(\mathrm{n}=12)$, unlikely to be admitted for more than 3 days $(\mathrm{n}=36)$, learning disability $(\mathrm{n}=6)$, presented unsafe behaviour $(n=12)$, did not speak English $(n=3)$, not available to meet $(n=18)$, delayed discharge $(\mathrm{n}=2)$

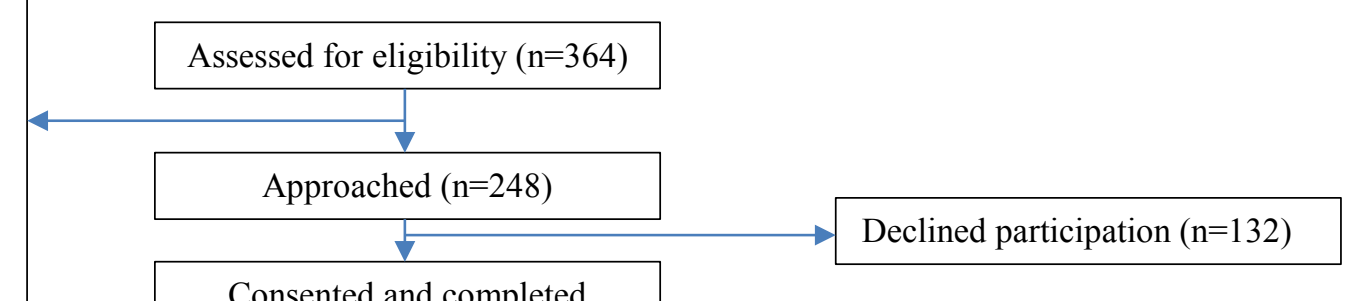

Excluded after giving consent for violating inclusion/exclusion criteria: discharged or moved wards within 2 days of giving consent and completing baseline measures $(n=14)$

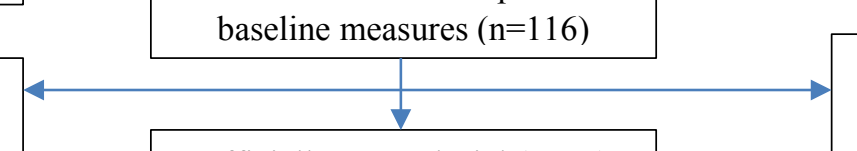

Excluded after giving consent for violating inclusion/exclusion criteria: discharged or moved wards within 2 days of giving consent and completing baseline measures $(n=6)$

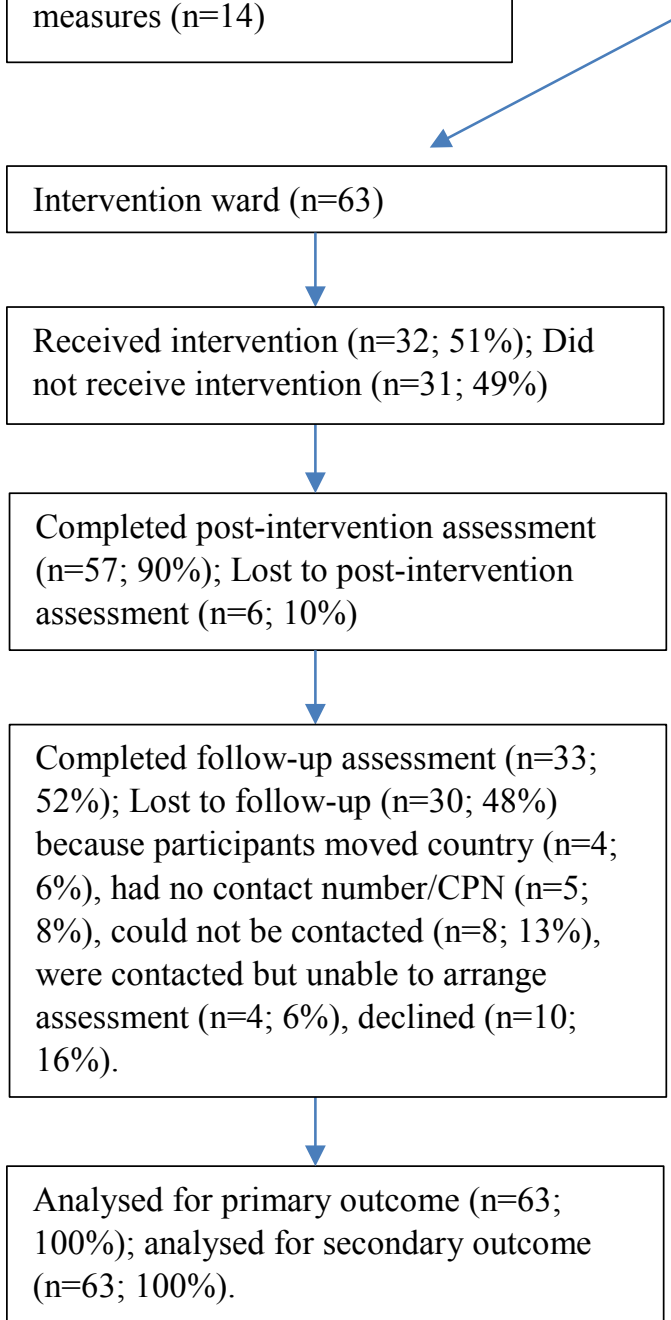

Control ward $(\mathrm{n}=33)$

Received TAU ( $\mathrm{n}=33 ; 100 \%)$; Did not receive TAU $(\mathrm{n}=0 ; 0 \%)$

Completed post-intervention assessment $(n=32$; 97\%); Lost to post-intervention assessment $(\mathrm{n}=1 ; 3 \%)$

Completed follow-up assessment $(\mathrm{n}=18 ; 55 \%)$; Lost to follow-up ( $\mathrm{n}=15 ; 45 \%)$ because participants died $(n=2 ; 6 \%)$, moved country $(\mathrm{n}=1 ; 3 \%)$, had not contact number/CPN $(\mathrm{n}=1$; $3 \%)$, could not be contacted $(n=1 ; 3 \%)$, declined $(n=5 ; 15 \%)$.

Figure 1: CONSORT diagram of participant flow 


\section{Appendices (online supplement)}

\section{Appendix $A$}

\section{Post-registration amendments}

Delivery of the psychological intervention as planned was challenging, suggesting that the model of intervention is in early stages of development and evaluation. Consequently, the aims and outcomes of the trial were re-specified according to the CONSORT guidelines for pilot and feasibility trials (Eldridge, Chan, et al., 2016) prior to analysis. The original aims of the study were to assess whether the addition of psychological intervention to TAU was more effective than TAU alone with regards to reducing readmissions, reducing psychological distress and increasing mental health related selfefficacy. However, in light of newly developed conceptual frameworks that define and guide reporting of pilot and feasibility trials (Eldridge, Chan, et al., 2016; Eldridge, Lancaster, et al., 2016; Lancaster, 2015) it became clear, during the course of this research, that the original aims and proposed methodology were ambitious and beyond the scope of this study. According to Eldridge, Lancaster, et al., (2016), the current study fitted under the umbrella term of 'feasibility studies'. As some aspects of a larger trial were also implemented in this study, e.g. gathering preliminary clinical outcome data (and predictor outcome data) and delivering the intervention, it may also fall into Eldridge, Lancaster, et al.'s (2016) subcategory of non-randomised pilot trials. However, as the primary aims are to determine the feasibility of delivering and evaluating the intervention in a future definitive trial, the current study is referred to as a feasibility study, therefore mapping onto phase two of the MRC framework for developing and evaluating complex interventions (Craig, 2012; Craig et al., 2008). Although changing the aims of a study can increases the risk of bias (Higgins \& Green, 2011), redefining the aims was deemed important for this study in order to inform a future, adequately powered, definitive trial of this intervention (Lancaster, 2015). Additionally, in reporting all amendments, the decision to make changes remains transparent and is therefore in keeping with the open science framework.

1. The aims and outcomes of the trial were re-specified according to the CONSORT guidelines for pilot and feasibility trials (Eldridge, Chan, et al., 2016) prior to analysis.

2. A supplementary per protocol analysis (i.e. excluding participants in the intervention group who did not receive the intervention) was included before analysis. A large proportion of participants in the intervention group did not receive the psychological intervention. Additionally, through direct observation, it was evident that ward staff involvement in co-facilitating and facilitating the groups was slow and in some cases absent. The ward psychologist facilitated group sessions where possible, however due to other commitments (i.e. individual sessions) delivery relied heavily on ward staff. Unfortunately, there was no protected time for ward staff to deliver groups, therefore given the busy nature of an acute inpatient ward staff few group therapies were delivered: only three group were delivered (rather than four), and some weeks no groups were delivered. Furthermore, a number of staff who attended relevant psychological training (i.e. CBT and MBT staff training) left the ward and were replaced by staff who had not attended psychological training. The absence of ward staff involvement in group delivery, along with few psychologically trained staff suggested culture change had not taken place yet and it was likely that a psychological milieu had not yet been adopted. This had clear implications for the clinical outcomes collected in the present study. For example, under the assumption that ward culture and environment had not changed (i.e. become more therapeutic) it is unlikely that inpatients in the intervention group who did not attend psychological groups or individual sessions would have benefited from the intervention. Thus, although pragmatic, an intention to treat analysis is likely to underestimate treatment effects (Dunn, 2013). For this reason, it was decided that the descriptive analysis in which all participants who formally entered the study are included (intention to treat), should be supplemented by a descriptive analysis in which participants in the intervention group who did not engage with the intervention are excluded (per treatment protocol).

3. Additional inclusion/exclusion criteria were added after recruitment began. Patients were not deemed eligible for participation if presenting unsafe behaviour, i.e. severe hostility/aggression 
or sexually uninhibited behaviour towards staff and participants were excluded if discharged or moved wards (to either the other participating ward or a non-participating ward) within two days of giving consent and completing baseline questionnaires.

4. Three groups ('Anxiety and Stress Management', 'Living Well with Emotions' and 'Being Friends with Yourself'), rather than four groups, were run when possible due to limited resource for group delivery.

5. In accordance with the change in study aims the sample size required for the current study was recalculated after the study began. The target sample size changed from 160 to 150 participants. 


\section{Appendix B}

\section{ADePT Process}

Using the ADePT process (Bugge et al., 2013), solutions to address problems identified by this trial were assessed. In addition to changes to the intervention, trial design and the clinical context, a fourth option was included where additional feasibility research was deemed necessary or informative before progressing to a full trial. As this option includes solutions which inform a future trial, rather than directly influence the trial or real world, no assessment of solutions in this category were carried out.

\section{Problem B1}

Type B: Poor implementation of group components of the intervention.

\section{Evidence}

- Few group therapies delivered overall and only 3 of 4 possible groups delivered during study period.

- Due to lack of staff engagement, i.e. no time, staff sickness, many staff with relevant psychological training left during study period, inflexible rotas and some staff did not feel confident in their ability to deliver groups.

- May have been influenced by poor integration of the intervention into the whole service.

- Influenced by above points and lack of support from managerial staff and psychiatrists.

All of the above may influence a trial and the real world.

\section{Solutions}

\section{Change aspects of:}

a) Intervention

1. Alter intervention, i.e. focus on fewer group types.

2. More thorough staff training.

b) Trial design

None

c) Clinical context

1. Increase psychological resource to increase psychological presence on the ward, psychological training and supervision for ward staff.

2. Flexible rotas, protected time for therapeutic work to ensure more consistent and frequent group interventions are delivered.

3. Hire staff specifically for group facilitator role.

4. Assign designated project 'champion'

d) Additional feasibility research

1. Qualitative work to investigate staff (from all levels) perception intervention acceptability

Assessment of solutions

and of barriers and facilitators to intervention implementation.

\section{a1: Alter intervention, i.e. focus on fewer group types.}

\section{Could a1 be effective in trial setting?}

Yes, likely to be less burden for service to release staff and may improve quality of delivery as fewer therapeutic approaches for staff to learn. However, dependent on $\mathrm{c} 1$ and $\mathrm{c} 2$ but the aims of the intervention should be reconsidered and the impact of the intervention may be diluted.

\section{Could a1 be feasible in trial setting?}

Likely to be more feasible than current protocol. However, dependent on $\mathrm{c} 1$ and support from management. Aims of the intervention will need to be reconsidered.

\section{Could al be effective in real world?}


Likely to be less burden for staff and improve quality of delivery. However, dependent on c1 and $\mathrm{c} 2$.

Could a1 be feasible in real world?

Likely to be more feasible than current protocol. However, dependent on c1 and support from management. Aims of the intervention would need to be reconsidered.

\section{a2: More thorough staff training.}

\section{Could a1 be effective in trial setting?}

Yes, likely to increase staff confidence in their ability to deliver groups, however dependent on c1.

Could al be feasible in trial setting?

Dependent on c1.

Could a1 be effective in real world?

Yes, likely to increase staff confidence in their ability to deliver groups, however dependent on c1.

Could a1 be feasible in real world?

Dependent on $\mathrm{c} 1$.

c1 Increase psychological resource to increase psychological presence on the ward, psychological training and supervision for ward staff.

\section{Could the solution be effective in trial setting?}

Yes, evidence of consistent delivery of groups when delivered by psychological team and cofacilitated by member of ward staff (Owen, Sellwood, Kan, Murray, \& Sarsam, 2015) but dependent on c2. 1:20 full time clinical psychologist to inpatient ratio is recommended by the Sainsbury Centre for Mental Health (2005). Additional time to co-facilitate more complex groups with staff will improve staff members' confidence in delivery.

\section{Could the solution be feasible in trial setting?}

Only if resource is available. Also dependent on c2.

\section{Could solution be effective in real world?}

Yes, evidence of consistent delivery of groups when delivered by psychological team and cofacilitated by member of ward staff (Owen et al., 2015) but dependent on c2. Additional time to co-facilitate more complex groups with staff will improve staff members' confidence in delivery.

\section{Could solution be feasible in real world?}

Yes, if cost-effective and psychological resource available. Also dependent on c2.

\section{c2 Flexible rotas, protected time for therapeutic work to ensure consistently delivered groups}

\section{Could the solution be effective in trial setting?}

Staff interested in facilitating groups should work 9-5 one day a week, additional to shifts, on the same day for a fixed period. This time can be used to facilitate and co-facilitate groups with psychologist (Hill, Clarke, \& Wilson, 2009).

\section{Could the solution be feasible in trial setting?}

Discussions with management required to establish this. Agreement from managerial staff to support staff to develop skills and facilitate groups. Management support has been identified as a facilitator to successful implementation of new interventions in similar contexts (Berry, Haddock, Kellett, Awenat, et al., 2016; Berry \& Haddock, 2008). 


\section{Could solution be effective in real world?}

Staff interested in facilitating groups could work 9-5 one day a week, additional to shifts, on the same day for a fixed period. This time could be used to facilitate and co-facilitate groups with psychologist (Hill et al., 2009).

Could solution be feasible in real world?

Yes, but dependent on organisational level factors, i.e. management and resource. Agreement from managerial staff to support staff to facilitate. Staff will need enthusiasm to agree to work an additional day.

\section{c3 Hire staff specifically for group facilitator role.}

\section{Could the solution be effective in trial setting?}

Yes, would ensure groups are delivered but does not conduce integration of the intervention into the whole service, therefore aims of the intervention would need to be reconsidered.

Could the solution be feasible in trial setting?

Unlikely that resource is available.

Could solution be effective in real world?

Yes, but does not conduce whole service approach, therefore aims of the intervention should be reconsidered.

Could solution be feasible in real world?

Unlikely that resource is available.

\section{c4 Assign designated project 'champion'}

\section{Could the solution be effective in trial setting?}

Yes, there is evidence that this technique is successfully used to improve intervention implementation (Shaw et al., 2012).

Could the solution be feasible in trial setting?

Dependant on the support received from staff members who are likely to be influential.

Could solution be effective in real world?

Yes, there is evidence that this technique is successfully used to improve intervention implementation (Shaw et al., 2012).

Could solution be feasible in real world?

Dependant on the support received from staff members who are likely to be influential. Discussions with management staff are required.

\section{Assessment of options and tolerance of trade-off between explanatory and pragmatic trial}

To improve delivery of group components, it may be more feasible to focus on fewer than four types initially. However, negotiations with management may still be required to protect time for delivery of even one group and the aims and reach of the intervention may need to be reconsidered. Alternatively, a designated member of staff might be employed as group facilitator, however it is unlikely that resource would be available and this solution is not conducive to integrating the intervention into the whole service, therefore the aims will again need to be reconsidered. Solutions that are more akin to the aims of intervention, i.e. to improve staff knowledge and skills in relation to CBT and increasing the reach of psychological therapy within service users and staff, are to improve the flexibility of nursing staff rotas and ensure they have protected time for group facilitation and to increase the psychological resource for more thorough staff training. The former may involve identifying staff who are interested in facilitating groups and are willing to work one nine-five day a week on the same day for a fixed period. This time can be used to facilitate and co-facilitate groups with psychologist (Hill et al., 2009). To achieve this, either staff my need to work an additional day, or managerial staff must allow a degree of flexibility in rotas. Both options are likely to be effective in terms of improving implementation of groups therapies, 
however knowledge of whether staff are willing to work additional hours to develop their skills or whether managerial staff will support routine delivery of groups by staff is lacking.

\section{Problem B2}

Type B: Problem with patient engagement with the intervention.

\section{Evidence}

- Only half the participants engaged.

- Few patients per group.

- Engagement may have been influenced by poor implementation of some components of the intervention (see poor implementation section).

- May have been influenced by poor integration of the intervention into the whole service.

- Engagement may have been influenced by broad inclusion criteria, i.e. including patients who do not want to receive the intervention.

All of the above may influence a future trial and the real world.

\section{$\underline{\text { Solutions }}$}

\section{Change aspects of:}

a) Intervention None

b) Trial design

1. Alter eligibility criteria to exclude patients that do not want to receive psychological intervention during their admission.

c) Clinical context

1. Increase psychological resource to make intervention more available, increase psychological presence on the ward and increase psychological training and supervision for ward staff (see solution c1 of problem B1).

2. Improve staff engagement with intervention (see problem B1).

d) Additional feasibility research

1. Qualitative work to investigate intervention acceptability and why patients do and do not want to receive psychological input during acute admission.

\section{Assessment of solutions}

b1 Alter eligibility criteria to exclude patients that do not want to receive psychological intervention during their admission, e.g. use a rating scale in initial consent meeting.

\section{Could the solution be effective in trial setting?}

Yes. Likely to reduce number of participants included in the trial who do not want to receive the intervention, but such a design ignores impact of therapeutic milieu.

\section{Could the solution be feasible in trial setting?}

Yes. Researcher would ask patients whether they want to receive psychological therapy during admission when patient is initially approached for the study.

\section{Could solution be effective in real world?}

Yes, if psychological therapy was routinely offered patients would have the option to receive it. Could solution be feasible in real world?

Yes, if psychological therapy was routinely offered patients would have the option to receive it.

c1 Increase psychological resource to make intervention more available, increase psychological presence on the ward and increase psychological training and supervision for ward staff. 
Could the solution be effective in trial setting?

Yes, 1:20 full time clinical psychologist to inpatient ratio recommended by (Sainsbury Centre for Mental Health, 2005). More time for staff training: staff education recommended to promote awareness of therapeutic principles as staff play a key role in encouraging and enabling participant attendance (Jacobsen, Morris, Johns, \& Hodkinson, 2011).

Could the solution be feasible in trial setting?

Yes, if budget allowed, however challenges of staff attendance would remain.

Could solution be effective in real world?

Staff education recommended to promote awareness of therapeutic principles as staff play a key role in encouraging and enabling patient attendance (Jacobsen et al., 2011).

Could solution be feasible in real world?

Unlikely in a financially restricted service. Recognised by The British Psychological Society (2012) that 1:20 ratio is unrealistic but argue that 0.5 per ward (as recommended by (Royal College of Psychiatrists, 2010) is too little.

\section{Assessment of options and tolerance of trade-off between explanatory and pragmatic trial}

Patients expressing an interest in the intervention when asked if they want to receive it could be added to eligibility criteria. This option will reduce the proportion of non-engaging participants recruited to the trial and will mimic routine practice, if the intervention is implemented. Such data could also be used to identify characteristics of those who do and do not want to receive psychological intervention during their acute admission. In addition, increasing psychological resource should be effective in improving patient engagement for both the trial and the real world. Increased psychological resource will allow increased staff training, however agreement from management may be necessary in order to release staff for training. Increased staff training is recommended in psychiatric inpatient services to promote awareness of therapeutic principles as staff play a key role in encouraging and enabling participant attendance (Jacobsen et al., 2011), however financial restrictions may be a barrier.

\section{Problem A1}

Type A: Problem with excluding eligible patients and initially including non-eligible patients.

\section{Evidence}

- $26 \%$ excluded if ward staff anticipated admission should be short.

- $\quad 15 \%$ patients excluded after consenting and completing baseline measures.

The above issues may influence a future trial.

\section{$\underline{\text { Solutions }}$}

\section{Change aspects of:}

a) Intervention

1. Provide therapy on an outpatient basis.

b) Trial design

1. Introduce two consent points. 1 to obtain initial consent and complete baseline measures. 2 to obtain consent to officially enter the trial 2 days after initial consent.

c) Clinical context None

\section{Assessment of Solutions}

\section{a1 Provide therapy on an outpatient basis}

Could the solution be effective in trial setting? 
Yes, short admissions would be less of a concern if patients could continue therapy after discharge, however the focus of the therapy may change.

Could the solution be feasible in trial setting?

The time of the end-point or post-intervention data collection point would need consideration for the control group. Logistics of doing so also needs consideration, e.g. whether resources are available to extend therapy to outpatients.

Could solution be effective in real world?

Yes, patients who have short admissions would still be eligible for therapy.

Could solution be feasible in real world?

Logistics of doing so also needs consideration, e.g. whether resources are available to extend therapy to outpatients. This seems unlikely given the already limited resource available.

b1 Introduce two consent points. 1 to obtain initial consent and complete baseline measures. 2 to obtain consent to officially enter the trial 2 days after initial consent.

\section{Could the solution be effective in trial setting?}

Yes, likely to reduce the number of eligible patients who are not recruited. Initially, baseline data obtained along with consent to do so. Then if still admitted at second time point, consent to enter the trial is sought. If the patient has been discharged by the second consent point they do not enter the trial.

Could the solution be feasible in trial setting?

Yes, if ethical approval obtained and trial has resource to introduce an extra meeting with participants. Recruitment is labour intensive.

Could solution be effective in real world?

N/A

Could solution be feasible in real world?

$\mathrm{N} / \mathrm{A}$

\section{$\underline{\text { Assessment of options and tolerance of trade-off between explanatory and pragmatic trial }}$}

Introducing two consent points could be effective in reducing the number of eligible patients that are excluded and ineligible patients that are included. Additional consent will need ethical approval and will require more time for data collection. Alternatively, therapy could continue to be received if patients were discharged quickly. However, given the limited resource already allocated to such services, it may be unlikely that therapy will be available after discharge.

\section{Problem A2}

\section{Type A: Poor completion of follow-up questionnaires.}

\section{Evidence}

- $\quad 52-53 \%$ of follow-up questionnaires completed.

- $\quad 20 \%$ of participants with missing follow-up questionnaires could not be contacted.

The above issues may influence a trial.

\section{Solutions}

Change aspects of:

a) Intervention

None

b) Trial design

1. At point of discharge, collect all contact details, alternative contact number and arrange appointment with participants. 
2. Increase contact with participant between discharge and follow-up, i.e. reminder letter/phone call.

3. Offer incentive.

4. Primary outcome should not rely on contacting participants at follow-up.

5. Create profile for individuals unlikely to complete follow-up assessments and develop more stringent eligibility criteria on that basis.

c) Clinical context

None

\section{Assessment of solutions}

b1: At point of discharge, collect all contact details and arrange appointment with participants.

Could the solution be effective in trial setting?

Yes, all current details are available to researcher therefore contacts lacking in medical files are no longer a problem.

Could the solution be feasible in trial setting?

Yes, can be stipulated in trial protocol. If patients can be met at post intervention this solution will be feasible. Completion of post-intervention assessments was good in this trial.

Could solution be effective in real world?

$\mathrm{N} / \mathrm{A}$

Could solution be feasible in real world?

$\mathrm{N} / \mathrm{A}$

b2: Increase contact with participant between discharge and follow-up, i.e. reminder letter/phone call.

Could the solution be effective in trial setting?

Technique used in other trials including acute inpatients (Jacobsen, Peters, \& Chadwick, 2016). Some evidence that increased phone calls improves completion in difficult to reach population (Kleschinsky, Bosworth, Nelson, Walsh, \& Shaffer, 2009)

Could the solution be feasible in trial setting?

Will require support from a research assistant per site.

Could solution be effective in real world?

N/A does not affect real world. Patient will not be contacted at follow-up in real world.

Could solution be feasible in real world?

N/A does not affect real world. Patient will not be contacted at follow-up in real world.

\section{b3: Offer incentive.}

Could the solution be effective in trial setting?

Some evidence to suggest monetary incentive improve completion of questionnaires (Brueton et al., 2014).

Could the solution be feasible in trial setting?

Payment offered to take part in research rather than receive clinical treatment is in line with guidance (Royal College of Physicians, 2007) but must have ethical approval.

Could solution be effective in real world?

Patient will not be contacted at follow-up in real world.

Could solution be feasible in real world?

Patient will not be contacted at follow-up in real world.

b4: Primary outcome should not rely on contacting participants at follow-up. 
Could the solution be effective in trial setting?

Good completion of outcomes collected via electronic database in this trial.

Could the solution be feasible in trial setting?

Easy and efficient method of data collection.

Could solution be effective in real world?

Patient will not be contacted at follow-up in real world.

Could solution be feasible in real world?

Patient will not be contacted at follow-up in real world.

\section{b5: Exclude patients on basis of meeting profile of participants likely to be lost to follow-up.}

Could the solution be effective in trial setting?

Likely to reduce missing data at follow-up (S. Eldridge \& Kerry, 2012).

Could the solution be feasible in trial setting?

Work to be done before trial to identify characteristics of those likely to be lost to follow-up.

Could solution be effective in real world?

Will compromise external validity in a main trial. No follow-up in real world, therefore may exclude participants that may benefit from intervention.

Could solution be feasible in real world?

Results may not apply to those likely to be missing follow-up data.

\section{Assessment of options and tolerance of trade-off between explanatory and pragmatic trial}

This is a pragmatic trial, therefore while excluding patients based on whether they are unlikely to provide follow-up data may reduce risk of bias associated with large proportions of missing data, it is not an option as trial results may not apply to a proportion of patients who may well benefit from the intervention (Eldridge \& Kerry, 2012).

As participants are met at discharge for assessment, it is recommended that collecting additional information (i.e. best contact details for follow-up) would solve the problem and fit best with the current protocol. Increased contact between discharge and follow-up will be useful and is a technique used in other trials including acute psychiatric inpatients (Jacobsen et al., 2016) and there is some evidence that increasing contact with patients improves outcome completion of difficult to reach participants (Kleschinsky et al., 2009). However, this solution will more time consuming. As incentives are commonly used and are effective in healthcare trials (Brueton et al., 2014; Royal College of Physicians, 2007) this may also be a helpful solution. 


\begin{tabular}{|c|c|c|c|c|c|c|}
\hline \multicolumn{7}{|c|}{ Appendix C } \\
\hline \multirow[b]{2}{*}{ Outcome } & No imputed data & \multicolumn{5}{|c|}{ Descriptive summary of follow-up questionnaire data by group where data is imputed and not imputed } \\
\hline & $\begin{array}{c}\text { mITT intervention } \\
\text { group (mean } \\
\text { SD) } \\
(\mathbf{n}=\mathbf{3 3})\end{array}$ & $\begin{array}{c}\text { Control } \\
\text { group } \\
\text { (mean } \\
\text { SD) } \\
(\mathbf{n}=18)\end{array}$ & $\begin{array}{c}\text { PP intervention } \\
\text { group (mean } \\
\text { SD) } \\
(\mathbf{n}=19)\end{array}$ & $\begin{array}{c}\text { mITT intervention } \\
\text { group (mean } \\
\text { SD) } \\
(\mathbf{n}=63)\end{array}$ & $\begin{array}{c}\text { Control } \\
\text { group } \\
\text { (mean } \\
\text { SD) } \\
(\mathbf{n}=33)\end{array}$ & $\begin{array}{c}\text { PP intervention } \\
\text { group (mean } \\
\text { SD) } \\
(\mathbf{n}=32)\end{array}$ \\
\hline CORE-10 & 15.64 & 17.50 & 15.38 & 15.70 & 17.59 & 15.44 \\
\hline Total & 7.38 & 8.56 & 7.58 & 7.38 & 8.56 & 7.47 \\
\hline CORE-10 & 10.25 & 10.78 & 10.48 & 10.30 & 10.82 & 10.52 \\
\hline Problems & 5.32 & 5.40 & 5.43 & 5.24 & 5.34 & 5.32 \\
\hline CORE-10 & 4.78 & 5.74 & 4.30 & 4.78 & 5.81 & 4.33 \\
\hline Functioning & 2.86 & 2.93 & 2.83 & 3.02 & 2.93 & 2.83 \\
\hline \multirow{2}{*}{ BSI-18 Total } & 24.04 & 26.17 & 24.52 & 24.05 & 26.30 & 24.59 \\
\hline & 11.99 & 14.02 & 12.84 & 12.06 & 14.02 & 12.95 \\
\hline BSI-18 & 4.86 & 5.32 & 4.92 & 4.88 & 5.30 & 4.96 \\
\hline Somatization & 3.89 & 4.08 & 4.19 & 3.89 & 4.02 & 4.19 \\
\hline BSI-18 & 10.19 & 10.21 & 10.28 & 10.14 & 10.29 & 10.20 \\
\hline Depression & 5.48 & 6.78 & 5.77 & 5.56 & 6.72 & 5.83 \\
\hline \multirow[t]{2}{*}{ BSI-18 Anxiety } & 9.00 & 10.64 & 9.33 & 9.03 & 10.71 & 9.44 \\
\hline & 5.64 & 5.97 & 5.66 & 5.56 & 6.03 & 5.76 \\
\hline \multirow[t]{2}{*}{ MHCS Total } & 59.23 & 54.20 & 59.69 & 59.24 & 54.18 & 59.78 \\
\hline & 12.86 & 15.22 & 11.88 & 12.62 & 15.05 & 11.82 \\
\hline \multirow[t]{2}{*}{ MHCS Optimism } & 22.21 & 20.16 & 22.70 & 22.17 & 20.17 & 22.64 \\
\hline & 5.71 & 6.55 & 5.09 & 5.64 & 6.38 & 4.98 \\
\hline \multirow[t]{2}{*}{ MHCS Coping } & 24.35 & 22.16 & 24.49 & 24.30 & 22.06 & 24.56 \\
\hline & 7.14 & 6.83 & 6.84 & 7.14 & 6.78 & 6.84 \\
\hline \multirow{2}{*}{ MHCS Advocacy } & 12.67 & 11.88 & 12.50 & 12.77 & 11.95 & 12.59 \\
\hline & 3.25 & 3.91 & 3.51 & 3.41 & 3.85 & 3.62 \\
\hline
\end{tabular}

Brief Symptom Inventory Scale (BSI); Clinical Outcomes Routine Evaluation (CORE); Mental Health Confidence Scale (MHCS); Modified intention to treat (mITT); Per protocol(PP); Standard deviation (SD). 
Summary of pre-intervention to follow-up change in questionnaire scores where no data is imputed

Pre to follow-up mean change (ITT analysis)

\begin{tabular}{|c|c|c|c|c|c|c|c|c|c|c|c|}
\hline \multirow[b]{2}{*}{ Outcome } & \multirow[b]{2}{*}{ Group } & \multicolumn{6}{|c|}{ Pre to follow-up mean change (ITT analysis) } & \multicolumn{4}{|c|}{$\begin{array}{l}\text { Pre to follow-up mean change (PP completer } \\
\text { analysis) }\end{array}$} \\
\hline & & $\mathbf{n}$ & Mean & SD & $\begin{array}{c}\mathrm{g} \\
(95 \% \mathrm{CI}) \\
\end{array}$ & Summary ${ }^{1}$ & $\mathbf{n}$ & Mean & SD & $\begin{array}{c}\mathrm{g} \\
(95 \% \mathrm{CI})\end{array}$ & Summary $^{1}$ \\
\hline \multirow[t]{2}{*}{ CORE-10 Total } & Intervention & 33 & -8.25 & 9.32 & \multirow[b]{2}{*}{$\begin{array}{c}-0.31 \\
(-0.89,0.27)\end{array}$} & \multirow{2}{*}{$\begin{array}{c}\text { Favouring } \\
\text { intervention } \\
\text { Small to medium } \\
\text { effect }\end{array}$} & 19 & -8.89 & 9.92 & \multirow[b]{2}{*}{$\begin{array}{c}-0.35 \\
(-1.00,0.30)\end{array}$} & \multirow[b]{2}{*}{$\begin{array}{l}\text { Favouring intervention } \\
\text { Small to medium effect }\end{array}$} \\
\hline & Control & 18 & -5.00 & 11.77 & & & 18 & -5.00 & 11.77 & & \\
\hline \multirow[t]{2}{*}{ CORE-10 Problems } & Intervention & 33 & -5.09 & 7.17 & \multirow{2}{*}{$\begin{array}{c}-0.05 \\
(-0.63,0.53) \\
\end{array}$} & \multirow{2}{*}{ No effect } & 19 & -4.95 & 6.78 & \multirow{2}{*}{$\begin{array}{c}-0.03 \\
(-0.68,0.61) \\
\end{array}$} & \multirow{2}{*}{ No effect } \\
\hline & Control & 18 & -4.72 & 7.78 & & & 18 & -4.72 & 7.78 & & \\
\hline \multirow{2}{*}{$\begin{array}{l}\text { CORE-10 } \\
\text { Functioning }\end{array}$} & Intervention & 33 & -1.88 & 3.67 & \multirow[b]{2}{*}{$\begin{array}{c}-0.64 \\
(-1.23,-0.05)\end{array}$} & \multirow{2}{*}{$\begin{array}{c}\text { Favouring } \\
\text { intervention } \\
\text { Medium to large } \\
\text { effect }\end{array}$} & 19 & -2.84 & 3.39 & \multirow[b]{2}{*}{$\begin{array}{c}-0.92 \\
(-1.60,-0.24)\end{array}$} & \multirow[b]{2}{*}{$\begin{array}{c}\text { Favouring intervention } \\
\text { Large effect }\end{array}$} \\
\hline & Control & 18 & 0.50 & 3.70 & & & 18 & 0.50 & 3.70 & & \\
\hline \multirow[t]{2}{*}{ BSI-18 Total } & Intervention & 33 & -15.19 & 21.91 & \multirow{2}{*}{$\begin{array}{c}-0.15 \\
(-0.73,0.43)\end{array}$} & \multirow{2}{*}{$\begin{array}{c}\text { Favouring } \\
\text { intervention } \\
\text { Very small effect }\end{array}$} & 19 & -16.00 & 24.18 & \multirow{2}{*}{$\begin{array}{c}-0.18 \\
(-0.83,0.47)\end{array}$} & \multirow{2}{*}{$\begin{array}{c}\text { Favouring intervention } \\
\text { Small effect }\end{array}$} \\
\hline & Control & 13 & -11.89 & 19.99 & & & 18 & -11.89 & 19.99 & & \\
\hline \multirow{2}{*}{$\begin{array}{l}\text { BSI-18 } \\
\text { Somatization }\end{array}$} & Intervention & 32 & -5.41 & 7.76 & \multirow{2}{*}{$\begin{array}{c}-0.20 \\
(-0.78,0.38)\end{array}$} & \multirow{2}{*}{$\begin{array}{c}\text { Favouring } \\
\text { intervention } \\
\text { Small effect }\end{array}$} & 19 & -6.11 & 7.80 & \multirow{2}{*}{$\begin{array}{c}-0.31 \\
(-0.96,0.34)\end{array}$} & \multirow{2}{*}{$\begin{array}{c}\text { Favouring intervention } \\
\text { Small effect }\end{array}$} \\
\hline & Control & 18 & -3.94 & 5.76 & & & 18 & -3.94 & 5.76 & & \\
\hline \multirow[t]{2}{*}{ BSI-18 Depression } & Intervention & 33 & -4.41 & 9.16 & \multirow{2}{*}{$\begin{array}{c}-0.01 \\
(-0.59,0.57) \\
\end{array}$} & \multirow{2}{*}{ No effect } & 19 & $-5.11)$ & 10.59 & & Favouring intervention \\
\hline & Control & 18 & -4.33 & 10.59 & & & 18 & -4.33 & 10.59 & $(-0.72,0.56)$ & No to very small effect \\
\hline BSI-18 Anxiety & Intervention & 33 & -5.94 & 9.08 & -0.28 & Favouring & 19 & -5.21 & 8.87 & -0.20 & Favouring intervention \\
\hline & Control & 18 & -3.56 & 7.04 & $(-0.86,0.30)$ & $\begin{array}{l}\text { 1ntervention } \\
\text { Small effect }\end{array}$ & 18 & -3.56 & 7.04 & $(-0.85,0.45)$ & Small effect \\
\hline MHCS Total $^{2}$ & Intervention & 33 & 9.03 & 21.39 & & Favouring & 19 & 14.16 & 23.45 & & \\
\hline & Control & 18 & -5.83 & 28.43 & $\begin{array}{c}0.61 \\
(0.02,1.20)\end{array}$ & $\begin{array}{c}\text { intervention } \\
\text { Medium to large } \\
\text { effect }\end{array}$ & 18 & -5.83 & 28.43 & $\begin{array}{c}0.75 \\
(0.09,1.42)\end{array}$ & $\begin{array}{l}\text { Favouring intervention } \\
\text { Large effect }\end{array}$ \\
\hline MHCS Optimism $^{2}$ & Intervention & 33 & 1.84 & 9.10 & 0.54 & Favouring & 19 & 3.11 & 10.55 & 0.59 & Favouring intervention \\
\hline & Control & 18 & -3.72 & 11.99 & $(-0.05,1.12)$ & Medium effect & 18 & -3.72 & 11.99 & $(-0.07,1.25)$ & Medium effect \\
\hline MHCS Coping $^{2}$ & Intervention & 33 & 3.91 & 10.37 & 0.42 & Favouring & 19 & 5.32 & 11.11 & 0.51 & Favouring intervention \\
\hline & Control & 18 & -1.00 & 13.11 & $(-0.16,1.00)$ & intervention & 18 & -1.00 & 13.11 & $(-0.07,1.25)$ & Medium effect \\
\hline
\end{tabular}


Appendix D

Summary of pre-intervention to follow-up change in questionnaire scores where no data is imputed

Pre to follow-up mean change (ITT analysis)

Pre to follow-up mean change (PP completer

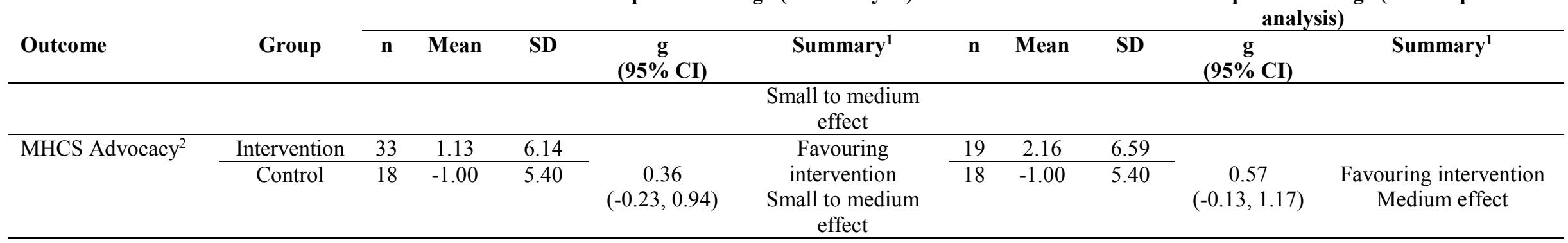

Brief Symptom Inventory Scale (BSI); Clinical Outcomes Routine Evaluation (CORE); Mental Health Confidence Scale (MHCS); Intention to treat (ITT); Per protocol(PP); Standard deviation (SD).

1. Summary is based on Cohen's (1988) benchmarks.

2. Positive effect favours intervention. 
Appendix $E$

\section{CONSORT 2010 checklist of information to include when reporting a pilot or feasibility trial*}

\begin{tabular}{|c|c|c|c|}
\hline Section/Topic & $\begin{array}{c}\text { Item } \\
\text { No }\end{array}$ & Checklist item & $\begin{array}{c}\text { Reported } \\
\text { on page } \\
\text { No }\end{array}$ \\
\hline \multicolumn{4}{|c|}{ Title and abstract } \\
\hline & $1 \mathrm{a}$ & Identification as a pilot or feasibility randomised trial in the title & p. 1 \\
\hline & $1 \mathrm{~b}$ & $\begin{array}{l}\text { Structured summary of pilot trial design, methods, results, and conclusions (for specific guidance } \\
\text { see CONSORT abstract extension for pilot trials) }\end{array}$ & p. 2 \\
\hline \multicolumn{4}{|l|}{ Introduction } \\
\hline \multirow[t]{2}{*}{$\begin{array}{l}\text { Background and } \\
\text { objectives }\end{array}$} & $2 a$ & $\begin{array}{l}\text { Scientific background and explanation of rationale for future definitive trial, and reasons for } \\
\text { randomised pilot trial }\end{array}$ & pp. 3-4 \\
\hline & $2 b$ & Specific objectives or research questions for pilot trial & pp. 4-5 \\
\hline \multicolumn{4}{|l|}{ Methods } \\
\hline \multirow[t]{2}{*}{ Trial design } & $3 a$ & Description of pilot trial design (such as parallel, factorial) including allocation ratio & p. 4 \\
\hline & $3 b$ & $\begin{array}{l}\text { Important changes to methods after pilot trial commencement (such as eligibility criteria), with } \\
\text { reasons }\end{array}$ & $\begin{array}{l}\text { p. } 4 \text { and } \\
\text { Appendices }\end{array}$ \\
\hline \multirow[t]{3}{*}{ Participants } & $4 a$ & Eligibility criteria for participants & pp. 5-6 \\
\hline & $4 \mathrm{~b}$ & Settings and locations where the data were collected & pp. 5-6 \\
\hline & $4 c$ & How participants were identified and consented & pp. 5-6 \\
\hline
\end{tabular}




\begin{tabular}{|c|c|c|c|}
\hline Interventions & 5 & $\begin{array}{l}\text { The interventions for each group with sufficient details to allow replication, including how and when } \\
\text { they were actually administered }\end{array}$ & pp. 6-7 \\
\hline \multirow[t]{3}{*}{ Outcomes } & $6 a$ & $\begin{array}{l}\text { Completely defined prespecified assessments or measurements to address each pilot trial objective } \\
\text { specified in } 2 b \text {, including how and when they were assessed }\end{array}$ & pp. 4-5 \\
\hline & $6 b$ & $\begin{array}{l}\text { Any changes to pilot trial assessments or measurements after the pilot trial commenced, with } \\
\text { reasons }\end{array}$ & $\begin{array}{l}\text { p. } 4 \text { and } \\
\text { Appendices }\end{array}$ \\
\hline & $6 c$ & $\begin{array}{l}\text { If applicable, prespecified criteria used to judge whether, or how, to proceed with future definitive } \\
\text { trial }\end{array}$ & p. 4 \\
\hline \multirow[t]{2}{*}{ Sample size } & $7 a$ & Rationale for numbers in the pilot trial & p. 6 \\
\hline & $7 \mathrm{~b}$ & When applicable, explanation of any interim analyses and stopping guidelines & $\mathrm{N} / \mathrm{A}$ \\
\hline \multicolumn{4}{|l|}{ Randomisation: } \\
\hline \multirow{2}{*}{$\begin{array}{l}\text { Sequence } \\
\text { generation }\end{array}$} & $8 a$ & Method used to generate the random allocation sequence & $\mathrm{N} / \mathrm{A}$ \\
\hline & $8 b$ & Type of randomisation(s); details of any restriction (such as blocking and block size) & $\mathrm{N} / \mathrm{A}$ \\
\hline $\begin{array}{l}\text { Allocation } \\
\text { concealment } \\
\text { mechanism }\end{array}$ & 9 & $\begin{array}{l}\text { Mechanism used to implement the random allocation sequence (such as sequentially numbered } \\
\text { containers), describing any steps taken to conceal the sequence until interventions were assigned }\end{array}$ & $\mathrm{N} / \mathrm{A}$ \\
\hline Implementation & 10 & $\begin{array}{l}\text { Who generated the random allocation sequence, who enrolled participants, and who assigned } \\
\text { participants to interventions }\end{array}$ & p. 6 \\
\hline \multirow[t]{2}{*}{ Blinding } & $11 \mathrm{a}$ & $\begin{array}{l}\text { If done, who was blinded after assignment to interventions (for example, participants, care } \\
\text { providers, those assessing outcomes) and how }\end{array}$ & $\mathrm{N} / \mathrm{A}$ \\
\hline & $11 \mathrm{~b}$ & If relevant, description of the similarity of interventions & $\mathrm{N} / \mathrm{A}$ \\
\hline $\begin{array}{l}\text { Statistical } \\
\text { methods }\end{array}$ & 12 & Methods used to address each pilot trial objective whether qualitative or quantitative & p. 7 \\
\hline
\end{tabular}




\begin{tabular}{|c|c|c|c|}
\hline \multicolumn{4}{|l|}{ Results } \\
\hline \multirow{2}{*}{$\begin{array}{l}\text { Participant flow } \\
\text { (a diagram is } \\
\text { strongly } \\
\text { recommended) }\end{array}$} & $13 a$ & $\begin{array}{l}\text { For each group, the numbers of participants who were approached and/or assessed for eligibility, } \\
\text { randomly assigned, received intended treatment, and were assessed for each objective }\end{array}$ & $\begin{array}{l}\text { p. } 7 \text { and } \\
\text { Figure } 1\end{array}$ \\
\hline & $13 b$ & For each group, losses and exclusions after randomisation, together with reasons & $\begin{array}{l}\text { p. } 8 \text { and } \\
\text { Figure } 1\end{array}$ \\
\hline \multirow[t]{2}{*}{ Recruitment } & $14 a$ & Dates defining the periods of recruitment and follow-up & p. 5 \\
\hline & $14 \mathrm{~b}$ & Why the pilot trial ended or was stopped & p. 5 and 6 \\
\hline Baseline data & 15 & A table showing baseline demographic and clinical characteristics for each group & Table 1 \\
\hline $\begin{array}{l}\text { Numbers } \\
\text { analysed }\end{array}$ & 16 & $\begin{array}{l}\text { For each objective, number of participants (denominator) included in each analysis. If relevant, } \\
\text { these numbers } \\
\text { should be by randomised group }\end{array}$ & $\begin{array}{l}\text { Figure } 1 \\
\text { and Tables } \\
3 \text { and } 4 .\end{array}$ \\
\hline $\begin{array}{l}\text { Outcomes and } \\
\text { estimation }\end{array}$ & 17 & $\begin{array}{l}\text { For each objective, results including expressions of uncertainty (such as } 95 \% \text { confidence interval) } \\
\text { for any estimates. If relevant, these results should be by randomised group }\end{array}$ & $\begin{array}{l}\text { pp. } 9-10, \\
\text { Table } 3 \text { and } \\
4\end{array}$ \\
\hline $\begin{array}{l}\text { Ancillary } \\
\text { analyses }\end{array}$ & 18 & Results of any other analyses performed that could be used to inform the future definitive trial & $\begin{array}{l}\text { pp. 7-10, } \\
\text { Appendices }\end{array}$ \\
\hline \multirow[t]{2}{*}{ Harms } & 19 & $\begin{array}{l}\text { All important harms or unintended effects in each group (for specific guidance see CONSORT for } \\
\text { harms) }\end{array}$ & p. 10 \\
\hline & $19 a$ & If relevant, other important unintended consequences & \\
\hline \multicolumn{4}{|l|}{ Discussion } \\
\hline Limitations & 20 & Pilot trial limitations, addressing sources of potential bias and remaining uncertainty about feasibility & p. 12 \\
\hline Generalisability & 21 & $\begin{array}{l}\text { Generalisability (applicability) of pilot trial methods and findings to future definitive trial and other } \\
\text { studies }\end{array}$ & pp. $10-12$ \\
\hline
\end{tabular}




\begin{tabular}{|l|c|l|l|l|}
\hline Interpretation & 22 & $\begin{array}{l}\text { Interpretation consistent with pilot trial objectives and findings, balancing potential benefits and } \\
\text { harms, and } \\
\text { considering other relevant evidence }\end{array}$ & pp. 10-12 \\
\hline & $22 a$ & Implications for progression from pilot to future definitive trial, including any proposed amendments & p. 14 & 4 \\
\hline Other information & 23 & Registration number for pilot trial and name of trial registry & p. 4 \\
\hline Registration & 24 & Where the pilot trial protocol can be accessed, if available & p. 4 & p. 4 \\
\hline Protocol & 25 & Sources of funding and other support (such as supply of drugs), role of funders & p. 4 \\
\hline Funding & 26 & Ethical approval or approval by research review committee, confirmed with reference number & \\
\hline
\end{tabular}

Citation: Eldridge SM, Chan CL, Campbell MJ, Bond CM, Hopewell S, Thabane L, et al. CONSORT 2010 statement: extension to randomised pilot and feasibility trials. BMJ. 2016;3555 